\title{
Quotient groups of the fundamental groups of certain strata of the moduli space of quadratic differentials
}

\author{
KATHARINE C WALKER
}

\begin{abstract}
In this paper, we study fundamental groups of strata of the moduli space of quadratic differentials. We use certain properties of the Abel-Jacobi map, combined with local surgeries on quadratic differentials, to construct quotient groups of the fundamental groups for a particular family of strata.
\end{abstract}

30F30; 14D20

\section{Introduction}

Little is currently known about the fundamental groups of strata of the moduli spaces of either abelian or quadratic differentials. In this paper we construct a quotient group of the fundamental group for a certain family of strata of quadratic differentials. We do so by mapping a stratum into a larger configuration space of points on surfaces and showing that the image of the fundamental group of the stratum under this map is in the kernel of a version of the Abel-Jacobi map. We then construct a set of generators for the kernel of the Abel-Jacobi map and show that in some cases the image of the fundamental group of the stratum in the fundamental group of the configuration space is equal to this kernel.

More specifically, let $\mathcal{Q}_{g}$ be the space of quadratic differentials over Teichmüller space $\mathcal{T}_{g}$ and let $\lambda=\left(k_{1}, \ldots, k_{n}\right)$ be a partition of $4 g-4$. Define $\mathcal{Q}_{g}\left(k_{1}, \ldots, k_{n}\right)=$ $\mathcal{Q}_{\lambda}$ to be the subset of $\mathcal{Q}_{g}$ of quadratic differentials with $n$ zeroes of order $k_{1}, \ldots, k_{n}$. Let $\overline{\mathcal{Q}}_{g}$ and $\overline{\mathcal{Q}}_{\lambda}$ be the analogous spaces over moduli space, $\mathcal{M}_{g}$. We are interested in $\pi_{1}\left(\overline{\mathcal{Q}}_{\lambda}\right)$; however, when $\mathcal{Q}_{\lambda}$ and $\overline{\mathcal{Q}}_{\lambda}$ are both connected we have a short exact sequence

$$
1 \rightarrow \pi_{1}\left(\mathcal{Q}_{\lambda}\right) \rightarrow \pi_{1}\left(\overline{\mathcal{Q}}_{\lambda}\right) \rightarrow \Gamma_{g} \rightarrow 1
$$

where $\Gamma_{g}$ is the genus $g$ mapping class group. In many cases (although not all) both $\mathcal{Q}_{\lambda}$ and $\overline{\mathcal{Q}}_{\lambda}$ are connected, so we focus on proving results about $\pi_{1}\left(\mathcal{Q}_{\lambda}\right)$.

To do this we first embed $\mathcal{Q}_{\lambda}$ into a larger configuration space. In particular, to any partition $\lambda$ we associate a generalized symmetric group $S_{\lambda}$ that allows points of equal weights to be exchanged. For $M \in \mathcal{T}_{g}$ and $\lambda$ of length $n$, let $M^{[n]}$ denote the space of 
$n$ ordered distinct marked points on $M$, and let $\operatorname{Sym}^{\lambda}(M)$ denote $M^{[n]} / S_{\lambda}$. Define $\mathrm{Sym}_{g}^{\lambda}$ to be the associated bundle over $\mathcal{T}_{g}$, and $\mathrm{Pic}_{g}^{4 g-4}$ the bundle over $\mathcal{T}_{g}$ with fiber $\mathrm{Pic}^{4 g-4}(M)$, the Picard variety parametrizing line bundles on $M$ of degree $4 g-4$. Then we have the following maps:

$$
\mathcal{Q}_{\lambda} \stackrel{i}{\rightarrow} \operatorname{Sym}_{g}^{\lambda} \stackrel{\mathrm{AJ}}{\rightarrow} \mathrm{Pic}_{g}^{4 g-4}
$$

The first map is given by considering the zeroes of a quadratic differential as weighted marked points. The second map is the Abel-Jacobi map, given by mapping a divisor to its associated line bundle. The maps in (1) induce a sequence of maps:

$$
\pi_{1}\left(\mathcal{Q}_{\lambda}\right) \stackrel{i_{*}}{\rightarrow} \pi_{1}\left(\operatorname{Sym}_{g}^{\lambda}\right) \stackrel{\mathrm{AJ}_{*}}{\rightarrow} \pi_{1}\left(\mathrm{Pic}_{g}^{4 g-4}\right) \cong H_{1}(M, \mathbb{Z})
$$

We show that $\mathrm{AJ}_{*} \circ i_{*}: \pi_{1}\left(\mathcal{Q}_{\lambda}\right) \rightarrow H_{1}(\Sigma, \mathbb{Z})$ is trivial, so the image of $i_{*}$ will be in the kernel of $\mathrm{AJ}_{*}$. In the case where there are at least $O(\sqrt{g})$ zeroes of order 1 in $\lambda$, we are able to construct a set of generators for the kernel of $\mathrm{AJ}_{*}$.

Theorem 1.1 Let $\lambda=\left(k_{1}^{a}, k_{2}^{b_{2}}, \ldots, k_{m}^{b_{m}}\right)$, with exponents satisfying $\sum_{i=2}^{m} b_{i}=b$ and $a \geq(3+\sqrt{9+8(2 g+b-2)}) / 2$. Then the kernel of $\mathrm{AJ}_{*}: \pi_{1}\left(\operatorname{Sym}_{g}^{\lambda}\right) \rightarrow H_{1}(M, \mathbb{Z})$ is generated by transpositions of zeroes of equal weight, squares of transpositions of zeroes of unequal weight, moving sets of points of equal weight opposite ways around generators of $\pi_{1}(M)$, and in some cases moving single points around homologically trivial curves.

A more precise statement of Theorem 1.1 is given in Section 4. To show that the elements detailed in Theorem 1.1 are contained in $\pi_{1}\left(\mathcal{Q}_{\lambda}\right)$ we first follow a variety of authors, including Eskin, Masur and Zorich [3], Lanneau [8] and Kontsevich and Zorich [6], to create local surgeries on surfaces with quadratic differentials that affect the flat metric induced by the quadratic differential in only a small area around a zero. We then take advantage of the fact that for any genus 0 stratum, $\mathcal{Q}_{\lambda} \cong \operatorname{Sym}^{\lambda}\left(\mathbb{P}^{1}\right.$ ) (in other words, a quadratic differential on $\mathbb{P}^{1}$ may have zeroes at any set of points) to create explicit curves of quadratic differentials in the hyperelliptic loci of certain strata. This leads to the following theorem:

Theorem 1.2 Let $\lambda=\left(1^{a}, k_{1}, \ldots, k_{n}\right)$ with $a>\max \left\{g+5, k_{1}, \ldots, k_{n}\right\}$, all $k_{i}$ even, and some $k_{i}=k_{j}, 1 \leq i, j \leq n$. Then $\operatorname{im}\left(i_{*}: \pi_{1}\left(\mathcal{Q}_{\lambda},(M, q)\right) \rightarrow \pi_{1}\left(\operatorname{Sym}_{g}^{\lambda}\right)\right)=$ $\operatorname{ker}\left(\mathrm{AJ}_{*}\right)$.

Theorem 1.2 states that for certain $\lambda$ we can describe the image of $\pi_{1}\left(\mathcal{Q}_{\lambda}\right)$ in $\pi_{1}\left(\operatorname{Sym}_{g}^{\lambda}\right)$. However, the kernel of $\pi_{1}\left(\mathcal{Q}_{\lambda}\right) \rightarrow \pi_{1}\left(\operatorname{Sym}_{g}^{\lambda}\right)$ may be nontrivial, so we have created a quotient group of $\pi_{1}\left(\mathcal{Q}_{\lambda}\right)$. 
The structure of the paper is as follows. In Section 2 we give some general background. In Section 3 we collect some results about $\pi_{1}\left(\operatorname{Sym}_{g}^{\lambda}\right)$, and in Section 4 we construct a set of generators for the kernel of $\mathrm{AJ}_{*}$ when $\lambda$ has sufficiently many zeroes of the same order. In Section 5 we recall some local surgeries that allow us alter the flat metric associated to a quadratic differential near a particular zero. In Section 6 and Section 7 we use the results of Section 5 to construct explicit elements in the $\operatorname{ker}\left(\mathrm{AJ}_{*}\right)$. Section 8 summarizes when we have the image of $\pi_{1}\left(\mathcal{Q}_{\lambda}\right) \rightarrow \pi_{1}\left(\operatorname{Sym}_{g}^{\lambda}\right)$ equal to the kernel of $\mathrm{AJ}_{*}: \pi_{1}\left(\operatorname{Sym}_{g}^{\lambda}\right) \rightarrow H_{1}(M, \mathbb{Z})$. Theorem 1.1 and Theorem 1.2 are proved in Section 4 and Section 8, respectively.

\section{Preliminary definitions}

A meromorphic quadratic differential, $q$, on a Riemann surface, $M$, is a meromorphic section of the square of the canonical bundle, $K_{M}$, of $M$. In local coordinates $q$ assigns to each $\left(U_{\alpha}, z_{\alpha}\right)$ a meromorphic function $f_{\alpha}$ such that

$$
f_{\beta}\left(z_{\beta}\right)\left(\frac{d z_{\beta}}{d z_{\alpha}}\right)^{2}=f_{\alpha}\left(z_{\alpha}\right), \quad d z_{\beta}=\frac{d}{d z_{\alpha}} z_{\beta} d z_{\alpha}
$$

on $U_{\alpha} \cap U_{\beta}$.

A horizontal trajectory, or simply a trajectory, of a quadratic differential $q$ on $M$ is a smooth curve $\gamma:[0,1] \rightarrow M$ such that $f(\gamma(t))\left(\gamma^{\prime}(t) d t\right)^{2}$ is real and positive for all $t$. Similarly, a vertical trajectory of $q$ is $\gamma:[0,1] \rightarrow M$ such that $f(\gamma(t))\left(\gamma^{\prime}(t) d t\right)^{2}$ is real and negative, and a $\theta$-trajectory is $\gamma$ such that the argument of $f(\gamma(t))\left(\gamma^{\prime}(t) d t\right)^{2}$ is $2 \theta$. (For $q=d z^{2}$ on $\mathbb{C}$ these correspond to straight lines of angle $\theta$.) Through every regular point of $q$ there exist unique horizontal and vertical trajectories that are transverse. Near a zero of $q$ of order $n, p_{0}$, we can choose a local coordinate, $\zeta$, such that $\zeta=0$ at $p_{0}$ and $q=\zeta^{n} d \zeta^{2}$. Then the $n+2$ curves $\gamma(t)=t e^{i(2 \pi /(n+2)) k}$ for $k=0,1, \ldots, n+1$, are all horizontal trajectories that dead-end into $p_{0}$. A $\theta$-trajectory between two critical points of $q$ is called a saddle connection.

Any nonzero quadratic differential on $M$ gives us a metric on $M$

$$
|\gamma|_{q}=\int_{\gamma}|f(z)|^{1 / 2}|d z|
$$

for $\gamma$ a real curve on $M$. Geodesics in this metric are unions of $\theta$-trajectories, with vertices at critical points of $q$.

Through much of this paper we will be concerned not just with individual quadratic differentials but also their moduli spaces. Let $\mathcal{T}_{g}$ be the Teichmüller space of a closed 
genus $g$ surface and $\mathcal{M}_{g}$ the genus $g$ moduli space. We consider the bundle $\mathcal{E}$ over $\mathcal{T}_{g}$ with fiber $H^{0}\left(M, K_{M}^{2}\right)$ over $M \in \mathcal{T}_{g}$, and define $\mathcal{Q}_{g}$ to be the subspace of the total space of $\mathcal{E}$ consisting of quadratic differentials that are not zero sections of $K_{M}^{2}$. Define $\mathcal{Q}_{g}\left(k_{1}, \ldots, k_{n}\right)$ to be the subspace of $\mathcal{Q}_{g}$ of quadratic differentials with zeroes of order $k_{1}, \ldots, k_{n}, \sum_{1}^{n} k_{i}=4 g-4$ and $k_{i} \in \mathbb{N}$. The set of integer partitions of $4 g-4$ give a natural stratification of $\mathcal{Q}_{g}$ and a single $\mathcal{Q}_{g}\left(k_{1}, \ldots, k_{n}\right)$ is often called stratum of $\mathcal{Q}_{g}$. We also occasionally consider an analog of $\mathcal{E}$ where each fiber is the space of meromorphic sections of $K^{2}$ with up to some fixed number of single poles, and then we can consider $\mathcal{Q}_{g}\left(k_{1}, \ldots, k_{n}\right)$ where some of the $k_{i}=-1$. All of these spaces are well known to be manifolds (see Veech [12], for example). $\mathcal{E}$ is also well-known to be the cotangent bundle of $\mathcal{T}_{g}$, and from this we see $\mathcal{Q}_{g}$ is a complex manifold of dimension $6 g-6$. Unless $n=1 \mathcal{Q}_{g}\left(k_{1}, \ldots, k_{n}\right)$ is not closed in $\mathcal{Q}_{g}$, as zeroes may collide to form higher order zeroes.

One can construct the analog of $\mathcal{E}$ over $\mathcal{M}_{g}, \overline{\mathcal{E}} \rightarrow \mathcal{M}_{g}$, again with fiber $H^{0}\left(\bar{M}, K_{\bar{M}^{2}}\right)$ over $\bar{M} \in \mathcal{M}_{g}$. Let $\overline{\mathcal{Q}}_{g}$ be the total space of $\overline{\mathcal{E}}$ minus zero sections. Alternatively, the mapping class group, $\Gamma_{g}$, acts on $\mathcal{Q}_{g}$ by a lift of its action on $\mathcal{T}_{g}$. We can also define $\overline{\mathcal{Q}}_{g}$ as the quotient of $\mathcal{Q}_{g}$ by $\Gamma_{g}$. Similarly define $\overline{\mathcal{Q}}_{g}\left(k_{1}, \ldots, k_{n}\right)$ as the quotient of $\mathcal{Q}_{g}\left(k_{1}, \ldots, k_{n}\right)$ by $\Gamma_{g}$. Since $\Gamma_{g}$ does not act freely, the $\overline{\mathcal{Q}}_{g}\left(k_{1}, \ldots, k_{n}\right)$ will be complex orbifolds.

In general we do not require the $k_{i}$ to be distinct, but if a stratum has multiple zeroes of the same order we will sometimes use the notation $\mathcal{Q}_{g}\left(k_{1}^{n}, k_{2}, \ldots, k_{n}\right)$ to indicate $\mathcal{Q}_{g}\left(k_{1}, k_{1}, \ldots, k_{1}, k_{2}, \ldots, k_{n}\right)$. We denote elements of $\mathcal{Q}_{g}\left(k_{1}, \ldots, k_{n}\right)$ as $(M, q)$ where $M$ is a Riemann surface together with a homeomorphism from a fixed genus $g$ surface to $M$, defined up to isotopy. When the specific orders of the zeroes are not important we will sometimes let $\lambda=\left(k_{1}, \ldots, k_{n}\right)$ denote a partition of $4 g-4$ and let $\mathcal{Q}_{\lambda}=\mathcal{Q}_{g}\left(k_{1}, \ldots, k_{n}\right)$. The length of $\lambda$ will be the number of $k_{i}$ in the partition.

The following sums up the structure of various strata:

Theorem 2.1 (Masur, Smillie, Veech) Every $\mathcal{Q}_{g}\left(k_{1}, \ldots, k_{n}\right)$ is nonempty, with four exceptions: $\mathcal{Q}_{1}(\varnothing), \mathcal{Q}_{1}(-1,1), \mathcal{Q}_{2}(3,1), \mathcal{Q}_{2}(4)$. With the exception of these four strata, the $\mathcal{Q}_{g}\left(k_{1}, \ldots, k_{n}\right)$ are complex manifolds of dimension $2 g-2+n$.

The same is true of the $\overline{\mathcal{Q}}_{g}\left(k_{1}, \ldots, k_{n}\right)$, except that they are orbifolds instead of manifolds. Proofs of the above may be found in Masur and Smillie [9] and Veech [12]. Genus 0 is special in that it is possible to construct a rational function, $f: \mathbb{P}^{1} \rightarrow \mathbb{P}^{1}$, with zeroes and poles wherever desired, and $f(z) d z^{2}$ is then a quadratic differential on $\mathbb{P}^{1}$. This implies that $\mathcal{Q}_{0}\left(k_{1}, \ldots, k_{n}\right)$ is simply the configuration space of $n$ points on the sphere, which is well-known to be connected. 
Proposition 2.2 Any $\mathcal{Q}_{0}\left(k_{1}, \ldots, k_{n}\right)$ is connected.

If one has a (ramified) cover of some $M$, one can pull back a quadratic differential on $M$ to get one on its cover.

Lemma 2.3 Let $\pi: \widetilde{M} \rightarrow M$ be a ramified double cover, $q$ a quadratic differential on $M$ and $\widetilde{q}$ its pullback under $\pi$. Let $\tilde{p}$ be a ramification point of $\pi$ and $p=\pi(\tilde{p})$. Then, if $p$ is a singularity of order $k$ of $q, \tilde{p}$ will be a singularity of order $2 k+2$ on $\tilde{q}$.

One can see this by noticing that a singularity of order $k$ corresponds to a cone angle of $(k+2) \pi$; doubling the cone angle at this singularity gives a cone angle of $2 k+4 \pi$ or a singularity of order $2 k+2$. More detailed proofs of Proposition 2.2 and Lemma 2.3 may be found in Lanneau [8].

Using double covers one may construct a continuous map between two different strata of quadratics differentials.

Construction 2.4 Let $\sum_{1}^{n} k_{i}=-4, n \geq 2 g+2$. We can construct a local map $\mathcal{Q}_{0}\left(k_{1}, \ldots, k_{n}\right) \rightarrow \mathcal{Q}_{g}\left(2 k_{1}+2, \ldots, 2 k_{2 g+2}+2, k_{2 g+3}^{2}, \ldots, k_{n}^{2}\right)$ by taking $\left(M^{\prime}, q^{\prime}\right) \in$ $\mathcal{Q}_{0}\left(k_{1}, \ldots, k_{n}\right)$ to its double cover ramified at its zeroes of order $k_{1}, \ldots, k_{2 g+2}$. This gives a surface $(M, q)$ of genus $g$ such that each of the first $2 g+2$ zeroes of order $k_{i}$ goes to one with order $2 k_{i}+2$, and we get 2 copies of each remaining zero.

For the above map to be global one would need to be able to distinguish between zeroes of the same order, so as to consistently choose ramification points.

Definition 2.5 Define a quadratic differential $(M, q) \in \mathcal{Q}_{g}\left(k_{1}, \ldots, k_{n}\right)$ to be hyperelliptic if $M$ is a hyperelliptic Riemann surface and $q$ is invariant under the hyperelliptic involution. Define $\mathcal{Q}_{g}\left(k_{1}, \ldots, k_{n}\right)$ to be hyperelliptic if it contains a hyperelliptic quadratic differential.

This definition of a hyperelliptic stratum is somewhat different from one frequently used in the literature, where a connected component of a stratum is called hyperelliptic if every quadratic differential in it is hyperelliptic.

Finally, since $1 \rightarrow \Gamma_{g} \rightarrow \mathcal{Q}_{\lambda} \rightarrow \overline{\mathcal{Q}}_{\lambda} \rightarrow 1$ is a fibration, if $\mathcal{Q}_{\lambda}$ and $\overline{\mathcal{Q}}_{\lambda}$ are both connected then $1 \rightarrow \pi_{1}\left(\mathcal{Q}_{\lambda}\right) \rightarrow \pi_{1}\left(\overline{\mathcal{Q}}_{\lambda}\right) \rightarrow \pi_{0}\left(\Gamma_{g}\right) \cong \Gamma_{g} \rightarrow 1$ will be short exact. Interestingly, not all of the $\overline{\mathcal{Q}}_{\lambda}$ are actually connected. For those that are disconnected, the connected components are classified by whether or not hyperelliptic quadratic differentials form a full-dimensional subset of the stratum. Lanneau proves this in [8]. 
Theorem 2.6 (Lanneau) For $g \geq 3$ the following strata have two connected components:

(1) $\overline{\mathcal{Q}}_{g}(4(g-k)-6,4 k+2), 0 \leq k \leq g-2$

(2) $\overline{\mathcal{Q}}_{g}\left((2(g-k)-3)^{2}, 4 k+2\right), 0 \leq k \leq g-1$

(3) $\overline{\mathcal{Q}}_{g}\left((2(g-k)-3)^{2}, 2 k+1^{2}\right),-1 \leq k \leq g-2$

where one is hyperelliptic and the other is not. For $g \geq 5$ all other strata have one component; for $g=3,4$ all other strata are connected with the following four exceptions: $\overline{\mathcal{Q}}_{3}(-1,9), \overline{\mathcal{Q}}_{3}(-1,3,6), \overline{\mathcal{Q}}_{3}(-1,3,3,3)$, and $\overline{\mathcal{Q}}_{4}(12)$. These four sporadic strata have two connected components, but neither is hyperelliptic. For $g=0,1$ all strata are connected, and for $g=2 \overline{\mathcal{Q}}_{2}(3,3,-1,-1)$ and $\overline{\mathcal{Q}}_{2}(6,-1,-1)$ have two components, but all others are connected.

In [13] we showed the following:

Theorem 2.7 Let $g \geq 2$. Then any stratum of the form $\mathcal{Q}_{g}\left(1^{g}, k_{1}, \ldots, k_{n}\right)$ is connected.

For $\lambda$ as in Theorem 2.7 both $\mathcal{Q}_{\lambda}$ and $\overline{\mathcal{Q}}_{\lambda}$ are connected, and $1 \rightarrow \Gamma_{g} \rightarrow \pi_{1}\left(\mathcal{Q}_{\lambda}\right) \rightarrow$ $\pi_{1}\left(\overline{\mathcal{Q}}_{\lambda}\right) \rightarrow 1$ is short exact.

\section{Surface braid groups}

In this section we collect some results about surface braid groups, to use in analyzing the kernel of $\mathrm{AJ}_{*}$.

Let $S_{n}$ be the standard symmetric group on $n$ letters. To any partition, $\lambda$, of $4 g-4$ we associate a symmetric group, $S_{\lambda}$, which allows equal values to be exchanged. For example, to $\left(1^{4}, 2,5^{2}\right)$ we associate $S_{4} \times S_{2}$. The length of $\lambda$ will be the number of elements it contains. For a particular $M \in \mathcal{T}_{g}$ and partition $\lambda$ of length $n$, let $M^{[n]}$ denote the space of $n$ ordered distinct marked points on $M$, and let $\operatorname{Sym}^{\lambda}(M)$ be $M^{[n]} / S_{\lambda}$.

$\pi_{1}\left(M^{[n]}\right)$ is well-known as the pure or special braid group of $n$ elements on a genus $g$ surface, which we will denote by $S B_{n}(M)$ or simply $S B_{n}$. Similarly, $\pi_{1}\left(M^{[n]} / S_{n}\right)$ is the full braid group on $M, B_{n}(M)$ or $B_{n}$. The generators of both $S B_{n}$ and $B_{n}$ are well-known. In particular, let $l_{1}, \ldots, l_{2 g}$ be $2 g$ standard generators of $\pi_{1}(M)$, let $\left(p_{1}, \ldots, p_{n}\right) \in M^{[n]}$, and let $\rho_{i j}, 1 \leq i \leq n, 1 \leq j \leq 2 g$, denote an element of $S B_{n}$ such that $p_{i}$ follows a path that is homotopic to $l_{j}$. Let $D$ be any disk containing 
$p_{k}, p_{l}, 1 \leq k<l \leq n$, and let $\kappa_{k l}$ be either generator of $\pi_{1}\left(D^{[2]},\left(p_{k}, p_{l}\right)\right)$. (We may extend this to an element of $\pi_{1}\left(M^{[n]}\right)$ by letting the other $n-2$ points move along constant paths.) Similarly, let $\sigma_{s t}$ be either generator of $\pi_{1}\left(D^{[2]} / S_{2},\left(p_{s}, p_{t}\right)\right)$. The following theorem is classical.

Theorem $3.1 S B_{n}$ is generated by the $\rho_{i j}, 1 \leq i \leq n, 1 \leq j \leq 2 g$, and the $\kappa_{k l}$, $1 \leq k<l \leq n . B_{n}$ is generated by the $\rho_{i j}$ and the $\sigma_{s(s+1)}, 1 \leq s<n$.

It should be noted that there are multiple nonequivalent ways to define each of the $\rho_{i j}$, $\kappa_{k l}$, and $\sigma_{s(s+1)}$; however, any choice yields a generating set.

Let $M_{m}$ denote $M-\left\{p_{n+1}, \ldots, p_{n+m}\right\}$, where the $p_{n+i}$ are any distinct points on $M$, and let $S B_{n, m}$ denote $S B_{n}\left(M_{m}\right)$ and $B_{n, m}$ denote $B_{n}\left(M_{m}\right)$. (We will only be concerned with the topology of $M_{m}$, which does not depend on the choice of $\left\{p_{n+1}, \ldots, p_{n+m}\right\}$.) Let $\kappa_{k l}, 1 \leq k \leq n, n<l \leq n+m$, denote $p_{k}$ moving in a simple loop around $p_{l}$. Again the generators of both $S B_{n, m}$ and $B_{n, m}$ are well-known.

Theorem 3.2 $S B_{n, m}$ is generated by the $\rho_{i j}$ and the $\kappa_{k l}, 1 \leq j \leq 2 g, 1 \leq i, k \leq n$, $1 \leq l \leq n+m . B_{n, m}$ is generated by the $\rho_{i j}$, the $\sigma_{s(s+1)}, 1 \leq s<n$, and the $\kappa_{k l}$, $1 \leq k \leq n, n<l \leq n+m$.

A reference for Theorem 3.1 and Theorem 3.2 is Birman [1].

We will primarily be interested in $\pi_{1}\left(\operatorname{Sym}^{\lambda}(M)\right)$, which we will denote by $B_{\lambda}(M)$ or $B_{\lambda}$. For $\lambda=\left(k^{n}\right), B_{\lambda}$ is just $B_{n}$. For more complicated $\lambda$ we note that the covering map $M^{[n]} \rightarrow \operatorname{Sym}^{\lambda}(M)$ is normal and thus

$$
S B_{n} \rightarrow B_{\lambda} \rightarrow S_{\lambda}
$$

is a short exact sequence. This tells us the generators of $B_{\lambda}$ :

Proposition 3.3 Let $\lambda$ be a partition of $4 g-4$ of length $n$. Then $B_{\lambda}$ is generated by the $\rho_{i j}, 1 \leq i \leq n, 1 \leq j \leq 2 g$, and for each pair $1 \leq k<l \leq n$, either $\sigma_{k l}$ if $p_{k}$ and $p_{l}$ are of the same weight, or $\kappa_{k l}$ if $p_{k}$ and $p_{l}$ are of different weights.

In fact it is possible to generate $B_{\lambda}$ with fewer transpositions; however, this generating set will suffice for our purposes. Although it will not be explicitly used in this paper, it is also worth noting that for particular choices of generating sets, the relations among the generators of $S B_{m}, S B_{n, m}$ and $B_{n}$ are well-known (see Scott [11], for example), and thus the same will be true for any of their subgroups.

Another classical result about surface braid groups is the following, from [4]:

Theorem 3.4 (Fadell-Neuwirth) The map $\left(M_{m}\right)^{[n]} \rightarrow\left(M_{m}\right)^{[r]}$ is a fibration, with fiber $\left(M_{m+r}\right)^{[n-r]}$. 
This fibration induces a long exact sequence of homotopy groups. All higher homotopy groups are trivial, so

$$
1 \rightarrow S B_{n-r, m+r}(M) \rightarrow S B_{n, m}(M) \rightarrow S B_{r, m}(M) \rightarrow 1
$$

is a short exact sequence.

Finally, we will sometimes want to distinguish the many different transpositions of two points on a surface. Let $p, p^{\prime} \in M$ and define an $e d g e, e$, to be an embedding of the interval $[0,1]$ in $M$ with endpoints $p$ and $p^{\prime}$. Let $U \subset M$ be a contractible neighborhood of $e$. Then we define $\sigma_{e}$ to be either of the two generators of $\pi_{1}\left(U^{[2]} / S_{2},\left(p, p^{\prime}\right)\right)$, with $\sigma_{e}^{-1}$ its inverse, and $\kappa_{e}$ to be either of the two generators of $\pi_{1}\left(U^{[2]},\left(p, p^{\prime}\right)\right)$ with $\kappa_{e}^{-1}$ its inverse. For a particular $(M, q)$, let $P=\left\{p_{1}, \ldots, p_{n}\right\}$ be the zeroes of $q$ and define $\bar{E}_{M, q}=\{e: I \hookrightarrow M \mid e(I) \cap P=e(0) \cup e(1)\}$ to be the set of all edges on $(M, q)$. We say $e \sim e^{\prime}$ if $e$ and $e^{\prime}$ are homotopic in $M \backslash P$ relative to their endpoints, and define $E_{M, q}=\bar{E}_{M, q} / \sim$. We index the set of all transpositions associated to $(M, q)$ by $E_{M, q}$.

\section{The kernel of the Abel-Jacobi map}

In this section we first define the Abel-Jacobi map, and then construct a set of generators for its kernel.

Define $\operatorname{Sym}_{g}^{\lambda}$ to be the bundle over $\mathcal{T}_{g}$ with fiber $\operatorname{Sym}^{\lambda}(M)$. Similarly let $\operatorname{Pic}^{4 g-4}(M)$ be the Picard variety parametrizing line bundles of degree $4 g-4$, and define $\mathrm{Pic}_{g}^{4 g-4}$ to be the bundle over $\mathcal{T}_{g}$ with fiber $\mathrm{Pic}^{4 g-4}(M)$. Let $\Lambda_{g}$ be the set of all integer partitions of $4 g-4$.

Then we have a sequence of maps:

$$
\mathcal{Q}_{\lambda} \stackrel{i}{\rightarrow} \mathrm{Sym}_{g}^{\lambda} \stackrel{\mathrm{AJ}}{\rightarrow} \mathrm{Pic}_{g}^{4 g-4}
$$

The first map is given by considering the zeroes of a quadratic differential as marked points, weighted by the order of the zero. The second map is the Abel-Jacobi map, given by mapping a divisor to its associated line bundle, $\operatorname{AJ}(D)=|D|$. The composition of these maps is fiber-wise trivial because every element of $\mathcal{Q}_{\lambda}$ over a particular $M \in \mathcal{T}_{g}$ maps to $K_{M}^{2} \in \mathrm{Pic}_{g}^{4 g-4}$.

Using the fact that $\operatorname{Pic}(M) \cong \operatorname{Jac}(M)$, the Jacobian variety of $M$, AJ may alternatively be defined (on each fiber of $\operatorname{Sym}_{g}^{\lambda}$ ) as a map $\operatorname{Sym}_{g}^{\lambda}(M) \rightarrow \operatorname{Jac}(M)$, defined as follows. 
Choose holomorphic 1 -forms $\omega_{i}, 1 \leq i \leq g$ such that the $\omega_{i}$ form a basis for $H^{0}\left(M, \Omega_{M}^{1}\right)$, and choose a basepoint $p_{0} \in M$. Then for $D=\sum_{1}^{n} k_{i} p_{i}$,

$$
\mathrm{AJ}(D)=\left(\sum_{1}^{n} k_{i} \int_{p_{0}}^{p_{i}} \omega_{1}, \ldots, \sum_{1}^{n} k_{i} \int_{p_{0}}^{p_{i}} \omega_{g}\right) .
$$

See Griffiths and Harris [5], for example, for the equivalence of these definitions of AJ. Since $\operatorname{Jac}(M)=H^{0}\left(M, K_{M}\right)^{*} / H_{1}(M, \mathbb{Z})$, there is a natural isomorphism $H_{1}(\operatorname{Jac}(M), \mathbb{Z}) \rightarrow H_{1}(M, \mathbb{Z})$. The Hurewicz map, $\pi_{1}(\operatorname{Jac}(M)) \rightarrow H_{1}(\operatorname{Jac}(M), \mathbb{Z})$, is an also an isomorphism in this case. From this perspective one can see that

$$
\mathrm{AJ}_{*}: \pi_{1}\left(\operatorname{Sym}_{g}^{\lambda}(M)\right) \rightarrow H_{1}(\operatorname{Jac}(M), \mathbb{Z}) \cong H_{1}(M, \mathbb{Z})
$$

takes a braid in $\pi_{1}\left(\operatorname{Sym}_{g}^{\lambda}(M)\right)$ to a union of homology classes of loops in $M$, where the loop followed by $p_{i}$ is weighted by $k_{i}$.

As previously noted it is possible that $\mathcal{Q}_{\lambda}$ is not connected, and in general we will denote a connected component of $\mathcal{Q}_{\lambda}$ by $\mathcal{Q}_{\lambda}^{0}$. Then the maps in (3) induce a sequence of maps:

$$
\pi_{1}\left(\mathcal{Q}_{\lambda}^{0}\right) \stackrel{i_{*}}{\rightarrow} \pi_{1}\left(\operatorname{Sym}_{g}^{\lambda}\right) \stackrel{\mathrm{AJ}_{*}}{\rightarrow} \pi_{1}\left(\mathrm{Pic}_{g}^{4 g-4}\right) \cong H_{1}(M, \mathbb{Z})
$$

$\left(\pi_{1}\left(\mathrm{Pic}^{4 g-4}(M)\right) \cong \pi_{1}(\operatorname{Jac}(M)) \cong H_{1}(M, \mathbb{Z})\right.$, and $\mathrm{Pic}_{g}^{4 g-4}$ is a bundle over a contractible space, so $\pi_{1}\left(\mathrm{Pic}_{g}^{4 g-4}\right) \cong H_{1}(M, \mathbb{Z})$ as well.) $\mathrm{AJ}_{*} \circ i_{*}$ will take the sum of the paths travelled by the zeroes of quadratic differentials to their corresponding homology class, where the paths are weighted by the order of the zero.

Since $\mathcal{T}_{g}$ is simply connected, if the map $\mathcal{Q}_{\lambda} \rightarrow \mathcal{T}_{g}$ has no empty fibers, then the image of $\mathcal{Q}_{\lambda}$ under $\mathrm{AJ} \circ i$ is also simply connected. In this case $\mathrm{AJ}_{*} \circ i_{*}$ will again be trivial.

It is not always true that $\mathcal{Q}_{\lambda} \rightarrow \mathcal{T}_{g}$ has no empty fibers; for example, there exist $\lambda$ for which the dimension of $\mathcal{Q}_{\lambda}$ is less than the dimension of $\mathcal{T}_{g}$. In this case we consider the inclusion $\iota: \mathcal{Q}_{\lambda} \hookrightarrow \mathcal{Q}_{g}$, and the induced map of their fundamental groups.
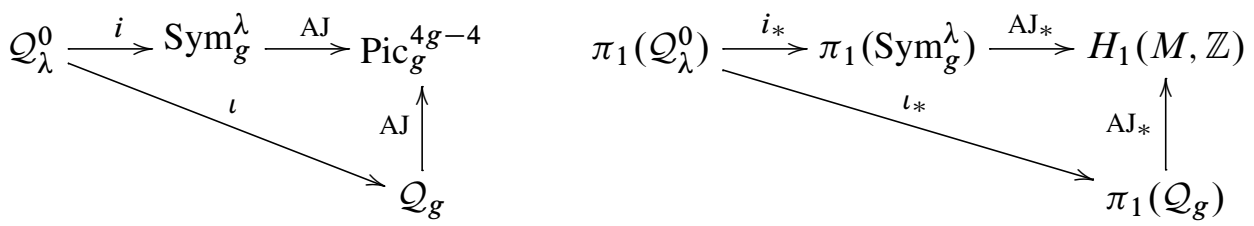

The diagram on the left clearly commutes, so the diagram on the right does as well. Since the image of $\mathcal{Q}_{g}$ under $\mathcal{Q}_{g} \rightarrow \mathcal{T}_{g}$ is indeed simply connected, we have $\mathrm{AJ}_{*} \circ \iota_{*}$ is trivial, and thus $\mathrm{AJ}_{*} \circ i_{*}$ is as well. The combination of these two cases gives us the following. 
Proposition 4.1 For all $\lambda \in \Lambda_{g}$ and any connected component $\mathcal{Q}_{\lambda}^{0}$ of $\mathcal{Q}_{\lambda}$

$$
\mathrm{AJ}_{*} \circ i_{*}: \pi_{1}\left(\mathcal{Q}_{\lambda}^{0}\right) \rightarrow H_{1}(M, \mathbb{Z})
$$

is trivial.

Proposition 4.1 implies that the image of $\pi_{1}\left(\mathcal{Q}_{\lambda}^{0}\right) \rightarrow \pi_{1}\left(\operatorname{Sym}_{g}^{\lambda}\right)$ will be in the kernel of $\mathrm{AJ}_{*}$. Since $\mathcal{T}_{g}$ is contractible, $\pi_{1}\left(\operatorname{Sym}_{g}^{\lambda}\right) \cong B_{\lambda}$. Thus, we now use the generators of $B_{\lambda}$ constructed in the previous section to create a set of generators for $\operatorname{ker}\left(\mathrm{AJ}_{*}: \pi_{1}\left(\operatorname{Sym}_{g}^{\lambda}\right) \rightarrow H_{1}(M, \mathbb{Z})\right)$. In Section 4.1 we consider $\lambda=\left(k^{n}\right)$, and in Section 4.2 more general $\lambda$.

\subsection{Strata with zeroes of only one weight}

We wish to calculate the kernel of $\mathrm{AJ}_{*}$ for $\lambda=\left(k^{n}\right)$ and $n$ reasonably large. Recall that we defined $M_{f}$ to be the surface $M$ with $f$ punctures. We have the following from [2]:

Theorem 4.2 (Copeland) If the surface $M$ has the structure of a polyhedron (a two-dimensional cell complex) of genus $g$ with $n$ vertices and $f$ faces such that the associated 1-skeleton has no double edges and no loops (edges with both ends at the same vertex $)$, then $\operatorname{ker}\left(\mathrm{AJ}_{*}: \pi_{1}\left(\operatorname{Sym}^{n}\left(M_{f}\right)\right) \rightarrow H_{1}(M, \mathbb{Z})\right)$ is generated by the edge set. Specifically the base point of $\operatorname{Sym}^{n}\left(M_{f}\right)$ may be chosen to be the vertices of the cell complex, each face may be viewed as having a puncture, and each edge may be viewed as a transposition of its two vertices.

Using the natural projection $\pi_{1}\left(\operatorname{Sym}^{n}\left(M_{f}\right)\right) \rightarrow \pi_{1}\left(\operatorname{Sym}^{n}(M)\right)$ one can show that the kernel of $\pi_{1}\left(\operatorname{Sym}^{n}(M)\right) \rightarrow H_{1}(M, \mathbb{Z})$ is a quotient group of the kernel of the map $\pi_{1}\left(\operatorname{Sym}^{n}\left(M_{f}\right)\right) \rightarrow H_{1}(M, \mathbb{Z})$ and is also generated by transpositions. Then Theorem 4.2 implies that if we can construct a graph with any number of faces on $M$ of the form described in the theorem, the kernel of $\mathrm{AJ}_{*}: \pi_{1}\left(\operatorname{Sym}^{n}(M)\right) \rightarrow H_{1}(M, \mathbb{Z})$ is generated by transpositions.

Copeland shows in [2] that it is possible to construct such a graph for any $g>2$ and $n=4 g-4$. We would like to show the same is true for smaller $n$, since $\lambda=\left(k^{n}\right)$ implies $n \leq 4 g-4$. In general, the best bound for $n$ we can hope to achieve will be on the order of $\sqrt{g}$. This is because a graph with $n$ vertices, no double edges, and no loops can have a maximum of $\left(\begin{array}{l}n \\ 2\end{array}\right)$ edges. The Euler characteristic then implies that

$$
2-2 g \leq n-\left(\begin{array}{l}
n \\
2
\end{array}\right)+f \text {. }
$$


Since $f$ must be $\geq 1$, at most $g$ grows at the rate of $n^{2}$. More specifically, solving the equation above we get $n \geq(3+\sqrt{9+8(2 g+f-2)}) / 2$. To show that graphs of the required form exist for $n$ close to this bound we will need some standard results from graph theory. Through the remainder of the section we will assume all graphs are connected, with no double edges or loops.

A 2-cell embedding of a graph, $G$, into a genus $g$ topological surface, $M_{g}$, is an embedding, $\varphi: G \rightarrow M_{g}$, such that each component of $M_{g} \backslash \varphi(G)$ is a 2-cell. The genus of $G, \gamma(G)$, is the minimal $g$ for which $G$ embeds into $M_{g}$ (such an embedding will always be a 2-cell embedding). The maximal genus of $G, \gamma_{M}(G)$, is the maximal $g$ for which $G$ has a 2-cell embedding into $M_{g}$. Let $v(G)$ and $e(G)$ denote the number of vertices and edges of $G$. For $\varphi$ a 2-cell embedding of $G$, let $f(G, \varphi)$ denote the number of components of $M_{g} \backslash \varphi(G)$, which we will call faces. Finally, let $K_{n}$ denote the complete graph on $n$ vertices. The following are well-known results in graph theory:

\section{Proposition 4.3 For any integer $n \geq 3$ :}

(1) $\gamma_{M}\left(K_{n}\right)=\left\lfloor\left(e\left(K_{n}\right)-v\left(K_{n}\right)+1\right) / 2\right\rfloor=\lfloor(n-2)(n-1) / 4\rfloor$.

(2) $\gamma\left(K_{n}\right)=\lceil(n-3)(n-4) / 12\rceil$.

(3) $K_{n}$ has a 2-cell embedding into $M_{g}$ if and only $\gamma\left(K_{n}\right) \leq g \leq \gamma_{M}\left(K_{n}\right)$.

See Kronk, Ringeisen and White [7], for example, for a survey of these results. Note that the maximal genus of $G$ is simply the largest genus for which $v(G)-\left(\begin{array}{c}v(G) \\ 2\end{array}\right)+f=2-2 g$ has a positive solution for $f$. A 2-cell embedding of $K_{n}$ into $M_{g}$ has $\left(\begin{array}{l}n \\ 2\end{array}\right)-n+2-2 g$ faces. We would like to show that graphs with a wider range of faces embed into $M_{g}$, and in fact knowing that $K_{n}$ 2-cell embeds into $M_{g}$ we can also show that "almost complete" graphs on $n$ vertices have 2-cell embeddings into $M_{g}$.

Lemma 4.4 If $K_{n}$ has a 2-cell embedding into $M_{g}$, then for any $f$ such that $1 \leq$ $f \leq\left(\begin{array}{l}n \\ 2\end{array}\right)-n+2-2 g$ there exists a graph with $n$ vertices that has a $2-c e l l$ embedding into $M_{g}$ with $f$ faces.

Proof We prove the lemma by induction on the number of faces. First, $K_{n}$ embeds into $M_{g}$ with $\left(\begin{array}{l}n \\ 2\end{array}\right)-n+2-2 g$ faces. Now suppose we have a connected graph $G$ that has a 2-cell embedding $\varphi: G \rightarrow M_{g}$ with $f(G, \varphi)$ faces, $2 \leq f(G, \varphi) \leq\left(\begin{array}{l}n \\ 2\end{array}\right)-n+2-2 g$. Each edge of a graph is adjacent to two faces (it may be adjacent to the same face twice), and since $G$ is connected any face must share at least one edge with some other face. Call this edge $e . G \backslash e$ will still be connected and $\left.\varphi\right|_{G \backslash e}$ will be a 2-cell embedding of $G \backslash e$ with one face fewer than $G$. 
Proposition 4.5 Let $1 \leq f \leq 4 g-4$. Then for any $n \geq(3+\sqrt{9+8(2 g+f-2)}) / 2$ there exists a graph embedded into a surface of genus $g$ with $n$ vertices, $f$ faces, no loops and no double edges.

Proof First, it suffices to show that for $n=\lceil(3+\sqrt{9+8(2 g+f-2)}) / 2\rceil$ there exists such a graph, $G$; to construct such a graph for $n^{\prime}>n$ we simply subdivide the edges of $G$ with the required number of additional vertices.

To prove the proposition for $n=\lceil(3+\sqrt{9+8(2 g+f-2)}) / 2\rceil$ note that

$$
\frac{3+\sqrt{9+8(2 g+f-2)}}{2} \leq n \leq \frac{3+\sqrt{9+8(2 g+f-2)}}{2}+1
$$

which implies

$$
2 g+f \leq \frac{(2 n-3)^{2}-9}{8}+2, \quad 2 g+f \geq \frac{(2 n-5)^{2}-9}{8}+2 .
$$

Since $1 \leq f \leq 4 g-4$,

$$
g \leq \frac{\left((2 n-3)^{2}-9\right) / 8+2-1}{2}, \quad g \geq \frac{\left((2 n-5)^{2}-9\right) / 8+2+4}{6} .
$$

Simplifying, we see that if $n=\lceil(3+\sqrt{9+8(2 g+f-2)}) / 2\rceil$ and $1 \leq f \leq 4 g-4$,

$$
\frac{n^{2}-5 n+16}{12} \leq g \leq \frac{(n-1)(n-2)}{4} .
$$

Since $n$ is positive, $\left(n^{2}-7 n+12\right) / 12 \leq\left(n^{2}-5 n+16\right) / 12$, and by Proposition $4.3 K_{n}$ has a 2-cell embedding into $M_{g}$. If $n=(3+\sqrt{9+8(2 g+f-2)}) / 2$, the embedding of $K_{n}$ has $f$ faces and is the desired graph. Otherwise the embedding of $K_{n}$ has $f^{\prime}$ faces, $f^{\prime}>f$, but then by Lemma 4.4 there exists a graph with $n$ vertices and an embedding into $M_{g}$ with $f$ faces.

Corollary 4.6 For $g \geq 2,1 \leq f \leq 4 g-4$ and $n \geq(3+\sqrt{9+8(2 g+f-2)}) / 2$, $\operatorname{ker}\left(\mathrm{AJ}_{*}: \pi_{1}\left(\operatorname{Sym}^{n}\left(M_{f}\right)\right) \rightarrow H_{1}(M, \mathbb{Z})\right)$ is generated by transpositions.

Proof This is an immediate consequence of Theorem 4.2 and Proposition 4.5.

Corollary 4.7 For $g \geq 2, n \geq(3+\sqrt{1+16 g}) / 2$ and $\lambda=\left(k^{n}\right)$,

$$
\operatorname{ker}\left(\mathrm{AJ}_{*}: \pi_{1}\left(\operatorname{Sym}_{g}^{\lambda}\right) \rightarrow H_{1}(M, \mathbb{Z})\right)
$$

is generated by transpositions. 
Proof Corollary 4.6 implies that $\operatorname{ker}\left(\mathrm{AJ}_{*}: \pi_{1}\left(\operatorname{Sym}^{n}\left(M_{1}\right)\right) \rightarrow H_{1}(M, \mathbb{Z})\right)$ is generated by transpositions. There is a natural projection $p: \pi_{1}\left(\operatorname{Sym}^{n}\left(M_{1}\right)\right) \rightarrow \pi_{1}\left(\operatorname{Sym}^{n}(M)\right)$; hence, $\operatorname{ker}\left(\mathrm{AJ}_{*}: \pi_{1}\left(\operatorname{Sym}^{n}(M)\right) \rightarrow H_{1}(M, \mathbb{Z})\right)$ may be viewed as a quotient group of $\operatorname{ker}\left(\mathrm{AJ}_{*}: \pi_{1}\left(\operatorname{Sym}^{n}\left(M_{1}\right)\right) \rightarrow H_{1}(M, \mathbb{Z})\right)$. This implies $\operatorname{ker}\left(\mathrm{AJ}_{*}: \pi_{1}\left(\operatorname{Sym}^{n}(M)\right) \rightarrow\right.$ $\left.H_{1}(M, \mathbb{Z})\right)$ is generated by products of transpositions. However, any single transposition in $\pi_{1}\left(\operatorname{Sym}^{n}\left(M_{1}\right)\right)$ is not in the kernel of $p$; thus, $\operatorname{ker}\left(\mathrm{AJ}_{*}: \pi_{1}\left(\operatorname{Sym}^{n}(M)\right) \rightarrow\right.$ $\left.H_{1}(M, \mathbb{Z})\right)$ is generated by transpositions.

Corollary 4.7 gives us the structure of the kernel of $\mathrm{AJ}_{*}$ for $\lambda=\left(k^{n}\right)$ and $n$ reasonably large.

When the length of $\lambda$ is small, it is more difficult to enumerate a set of generators for the kernel of $\mathrm{AJ}_{*}$. It is not true in general that the kernel will be generated by transpositions; for example, consider $i_{*} \mathcal{Q}_{g}(4 g-4) \subset \mathrm{Sym}_{g}^{1}$. We may move the single marked point around a curve that is homologically but not homotopically trivial. This will be in the kernel of $\mathrm{AJ}_{*}$ but is not a product of transpositions. However, for $\lambda$ with sufficiently many zeroes of the same order we may make some generalizations to the results of this subsection. In particular, a combination of Corollary 4.6 and the Fadell-Neuwirth fibration will allow us to generalize Corollary 4.7 to a larger class of $\lambda$, and this is what we will do in the next subsection.

\subsection{Strata with zeroes of more than one weight}

We would like to generalize Corollary 4.7 to $\lambda=\left(k_{1}^{n_{1}}, \ldots, k_{m}^{n_{m}}\right)$ with $n_{1}$ large. Let $\lambda$ be of length $n$ and let $\left(p_{1}, \ldots, p_{n}\right) \in \operatorname{Sym}_{g}^{\lambda}(M)$ be the basepoint of $B_{\lambda}$. From Section 3 we know that $B_{\lambda} \cong \pi_{1}\left(\operatorname{Sym}_{g}^{\lambda}\right)$ is generated by the $\rho_{i r}, 1 \leq i \leq n, 1 \leq r \leq 2 g$, and for each pair $1 \leq i<j \leq n$, either $\sigma_{i j}$ if $p_{i}$ and $p_{j}$ are of the same weight, or $\kappa_{i j}$ if $p_{i}$ and $p_{j}$ are of different weights. We can immediately show that some of these generators are in $\operatorname{ker}\left(\mathrm{AJ}_{*}: \pi_{1}\left(B_{\lambda}\right) \rightarrow H_{1}(M, \mathbb{Z})\right)$ :

Lemma 4.8 Any transposition or square transposition in $B_{\lambda}, \sigma_{i j}$ or $\kappa_{i j}$, is in the kernel of $\mathrm{AJ}_{*}$.

Proof A transposition of two points of equal weight consists of moving them in opposite directions along homotopic paths. The sum of these paths is then homotopic (and therefore homologous) to zero. A square transposition of two points of unequal weight moves each point along some path, and then back along a homologous path. Thus both points follow paths that are homologous to zero. 
An individual $\rho_{i r}$ will not be in the kernel of $\mathrm{AJ}_{*}$; however, there are two cases when it is easy to see that a product of them will be. First, if two sets of points of equal total weight follow $l_{r}$ and $l_{r}^{-1}$ respectively, then their paths will cancel each other out in $H_{1}(M, \mathbb{Z})$. Second, a single $p_{i}$ may follow a path that is homologically trivial but not homotopically trivial. More precisely, we have the following two definitions.

Definition 4.9 Fix $r, 1 \leq r \leq 2 g$. A null $\rho_{r}$ is a product $\prod_{n=1}^{m} \rho_{i_{n} r}^{ \pm 1}$ such that $\sum_{n=1}^{m} \pm k_{i_{n}}=0$, where the sign in front of $k_{i_{n}}$ is given by the sign of the exponent of $\rho_{i_{n} r}$.

Definition 4.10 Let $\lambda=\left(k_{1}, \ldots, k_{n}\right)$, fix $i, 1 \leq i \leq n$, and let $M_{n-i}$ be $M$ punctured at $p_{i+1}, \ldots, p_{n}$. An $i$-commutator is a product of $\rho_{i r}$ and $\kappa_{i j}, 1 \leq r \leq 2 g, i+1 \leq$ $j \leq n$, such that the path followed by $p_{i}$ is in $\left[\pi_{1}\left(M_{n-i}\right), \pi_{1}\left(M_{n-i}\right)\right]$.

In the remainder of the section we show that for $\lambda=\left(k_{1}^{n_{1}}, \ldots, k_{m}^{n_{m}}\right)$ with $n_{1}$ large, transpositions, square transpositions, null $\rho_{r}$, and $i$-commutators for $p_{i}$ not of order $k_{1}$ suffice to generate the kernel of $\mathrm{AJ}_{*}$. For $n_{2}, \ldots, n_{m}$ all sufficiently large, transpositions, square transpositions, and null $\rho_{r}$ suffice to generate.

To show this first recall that for any $\lambda$ of length $n$

$$
1 \rightarrow S B_{n} \rightarrow \pi_{1}\left(\mathrm{Sym}_{g}^{\lambda}\right) \cong B_{\lambda} \stackrel{\mathrm{pr}}{\rightarrow} S_{\lambda} \rightarrow 1
$$

is a short exact sequence. Thus for the remainder of the section we regard $S B_{n}$ as a natural subgroup of $B_{\lambda}$. From Section 3 we have that $S B_{n}$ is generated by $\rho_{i r}$ and $\kappa_{i j}, 1 \leq i<j \leq n, 1 \leq r \leq 2 g$.

Lemma 4.11 Any $Z \in B_{\lambda}$ can be written as $Y \cdot X$ where $Y$ is a product of transpositions and $X \in S B_{n}$.

Proof Let $\operatorname{pr}(Z)=\bar{Y} \in S_{\lambda}$. For every $p_{i}, p_{j}$ of the same weight, pick a transposition, $\sigma_{i j} \in B_{\lambda}$. Let $\operatorname{pr}\left(\sigma_{i j}\right)=\bar{\sigma}_{i j}$ and note that $S_{\lambda}$ is generated by the $\bar{\sigma}_{i j}$. Then we can write $\bar{Y}$ as a product of the $\bar{\sigma}_{i j}$, and we construct $Y \in B_{\lambda}$ by writing $Y$ as a product of the corresponding $\sigma_{i j}$. Then $\operatorname{pr}(Y)=\operatorname{pr}(Z)=\bar{Y}$. Let $X=Y^{-1} Z$. Since $\operatorname{pr}(X)=1$ and (5) is exact, $X \in S B_{n}$.

Let $K=\operatorname{ker}\left(\mathrm{AJ}_{*}: B_{\lambda} \rightarrow H_{1}(M, \mathbb{Z})\right)$. Then $K \cap S B_{n}=\operatorname{ker}\left(\mathrm{AJ}_{*}: S B_{n} \rightarrow H_{1}(M, \mathbb{Z})\right)$. By Lemma 4.11, to prove that any $Z \in K$ is a product of null $\rho_{r}, \sigma_{i j}, \kappa_{i j}$, and $i-$ commutators it suffices to show that this is true for all $X \in S B_{n}$. To show this we use the short exact sequence of Theorem 3.4:

$$
1 \rightarrow S B_{a, b} \rightarrow S B_{a+b}=S B_{n} \stackrel{\mathrm{pr}^{\prime}}{\rightarrow} S B_{b} \rightarrow 1
$$


for any $a, b$ such that $a+b=n$. Again we regard $S B_{a, b}$ as a natural subgroup of $B_{\lambda}$ for the remainder of the section. $K \cap S B_{a, b}=\operatorname{ker}\left(\mathrm{AJ}_{*}: S B_{a, b} \rightarrow H_{1}(M, \mathbb{Z})\right)$. Lemma 4.13 will prove that words in $K \cap S B_{a, b}$ can be written as a product of the desired elements, and Theorem 4.14 will then prove the same for words in $K \cap S B_{n}$ (and therefore $K$ ).

First we need a technical lemma, and in it we break up $S B_{a, b}$ further, using Theorem 3.4,

$$
1 \rightarrow S B_{a-1, b+1} \rightarrow S B_{a, b} \stackrel{\mathrm{pr}^{\prime \prime}}{\rightarrow} S B_{1, b} \rightarrow 1
$$

for the purpose of analyzing it.

Lemma 4.12 Let $\lambda=\left(k_{1}^{n_{1}}, \ldots, k_{m}^{n_{m}}\right)$, with $\sum_{1}^{m} n_{i}=n$. Pick any $a, 1 \leq a \leq n$, let $b=n-a$, and let $p_{a}$ have weight $k_{l}, 1 \leq l \leq m$. Then $\operatorname{im}\left(\mathrm{pr}^{\prime \prime}: K \cap S B_{a, b} \rightarrow S B_{1, b}\right)$ lies in $\left\{S \in S B_{1, b} \mid \mathrm{AJ}_{*}(S) \in d H_{1}(M, \mathbb{Z})\right\}$, where $d$ is the smallest positive integer such that there exist $c_{1}, c_{2}, \ldots, \widehat{c_{l}}, \ldots, c_{m} \in \mathbb{Z}$ such that $c_{1} \cdot k_{1}+c_{2} \cdot k_{2}+\cdots+d \cdot k_{l}+\cdots+c_{m} \cdot k_{m}=$ 0 has a solution.

Proof $S B_{a, b}$ is generated by $\rho_{i r}, \kappa_{i j}, 1 \leq i \leq a, 1 \leq j \leq a+b, 1 \leq r \leq 2 g$. We need not consider the $\kappa_{i j}$ as they all go to zero under $\mathrm{AJ}_{*}$. Let $w(i)$ be the weight of $p_{i}, w(i) \in\left\{k_{1}, \ldots, k_{m}\right\}$. Any word $W$ in $K \cap S B_{a, b}$ has the property that for a fixed $r$ the product of $\rho_{i r}^{ \pm 1}$ in $W$ must be such that $\sum \pm w(i)=0$. (The sign in front of $w(i)$ corresponds to the sign in the exponent of $\rho_{i r}$.) This implies that the number of times $\rho_{a r}$ occurs in a particular word must be a multiple of $d$.

Lemma 4.13 Let $\lambda, a$ and $b$ be as in Lemma 4.12. For all $X_{b} \in K \cap S B_{a, b}$ there exists $W \in K \cap S B_{a, b}$ such that $\operatorname{pr}^{\prime \prime}\left(X_{b}\right)=\operatorname{pr}^{\prime \prime}(W)$ and $W$ is a product of null $\rho_{r}$, $\kappa_{a j}$ or $\sigma_{a j}$, and $a$-commutators.

Proof As in Lemma 4.12 let $p_{a}$ be of weight $k_{l}$ and also let $c_{1}, c_{2}, \ldots, \widehat{c_{l}}, \ldots, c_{m}$, and $d$ be as in Lemma 4.12. $S B_{1, b}=\pi_{1}\left(M_{b}\right)$ is generated by $\bar{\rho}_{a r}, \bar{\kappa}_{a j}, a+1 \leq j \leq a+b$, $1 \leq r \leq 2 g$, where $\rho_{a r}, \kappa_{a j} \in S B_{a, b}$ project to their corresponding barred elements. (In $S B_{a, b}$ the points $p_{1}, \ldots, p_{a}$ move around punctures $p_{a+1}, \ldots, p_{a+b}$; under $\mathrm{pr}^{\prime \prime}, p_{a}$ moves around $p_{a+1}, \ldots, p_{a+b}$.) Let $G=\left[S B_{1, b}, S B_{1, b}\right]$ be the commutator of $S B_{1, b}$.

Since $G$ abelianizes $S B_{1, b}$, for all $X_{b} \in K \cap S B_{a, b}$ there exists $\bar{h} \in G$ such that $\bar{h} \cdot \operatorname{pr}^{\prime \prime}\left(X_{b}\right)$ is a product of $\bar{\rho}_{a r}, \bar{\kappa}_{a j}$, such that all $\bar{\kappa}_{a j}$ are on the right of all $\bar{\rho}_{a r}$ and if $r_{1}<r_{2}, \bar{\rho}_{a r_{1}}$ is to the left of $\bar{\rho}_{a r_{2}}$. In other words, $\bar{h} \cdot \operatorname{pr}^{\prime \prime}\left(X_{b}\right)$ is a word such that for fixed $r$ all $\bar{\rho}_{a r}$ are adjacent. By Lemma 4.12, the power of any $\bar{\rho}_{a r}$ in $\bar{h} \cdot \operatorname{pr}^{\prime \prime}(X)$ must be a multiple of $d$. 
Now we construct $W \in K \cap S B_{a, b}$ by inserting $c_{i}$ elements of weight $k_{i}$ moving around $l_{r}$ adjacent to each set of $d$ elements of weight $k_{l}$ moving around the $l_{r}$ in $\bar{h} \cdot \operatorname{pr}^{\prime \prime}\left(X_{b}\right)$, and then multiplying by $h^{-1}, \operatorname{pr}^{\prime \prime}(h)=\bar{h}$. For example, let $\lambda=\left(k_{1}, k_{2}\right)$ with $p_{1}$ of weight $k_{1}, p_{2}$ of weight $k_{2}$, and $c k_{1}-d k_{2}=0$. If $\bar{h} \cdot \operatorname{pr}^{\prime \prime}(X)=\bar{\rho}_{2 r_{1}}^{n_{1}} \bar{\rho}_{2 r_{2}}^{n_{2}} \bar{\kappa}_{12}$, then $W=h^{-1}\left(\rho_{2 r_{1}}^{n_{1}} \rho_{1 r_{1}}^{-(c / d) n_{1}}\right)\left(\rho_{2 r_{2}}^{n_{2}} \rho_{1 r_{2}}^{-(c / d) n_{2}}\right) \kappa_{12}$, where Lemma 4.12 implies $n_{1}$ and $n_{2}$ are divisible by $d$. Both of the elements in parentheses are null $\rho_{r} . W$ is thus made up of null $\rho_{r}, \kappa_{a j}$, and $a$-commutators. By construction $\operatorname{pr}^{\prime \prime}(W)=\operatorname{pr}^{\prime \prime}\left(X_{b}\right)$.

If there are sufficiently many points of weight $k_{l}$ (if $\left.n_{l} \geq(3+\sqrt{9+8(2 g+b-2)}) / 2\right)$, then by Corollary $4.6 \operatorname{ker}\left(\mathrm{AJ}_{*}: \pi_{1}\left(\operatorname{Sym}^{n_{l}}\left(M_{b}\right) \rightarrow H_{1}(M, \mathbb{Z})\right)\right.$ is generated by transpositions. This implies that the $a$-commutators that make up $h^{-1}$ above can be written as products of transpositions.

Theorem 4.14 Let $\lambda=\left(k_{1}^{a}, k_{2}^{b_{2}}, \ldots, k_{m}^{b_{m}}\right)$, with exponents satisfying $\sum_{k=2}^{m} b_{k}=b$ and $a \geq(3+\sqrt{9+8(2 g+b-2)}) / 2$ (as in Corollary 4.6). Then any $Z \in K$ can be written as a product of null $\rho_{r}, \sigma_{i j}, \kappa_{i j}, 1 \leq i, j \leq a+b, 1 \leq r \leq 2 g$, and $i$-commutators for $p_{i}$ not of weight $k_{1}$.

Proof By Lemma 4.11 it suffices to prove the theorem for all $X \in K \cap S B_{a+b}$. By Corollary 4.6 the theorem is true for all $X_{b} \in K \cap S B_{a, b}$.

For $O \leq k \leq b$ we have the following version of the short exact sequence in (7):

$$
1 \rightarrow S B_{a+k-1, b-k+1} \rightarrow S B_{a+k, b-k} \stackrel{\mathrm{pr}^{\prime \prime}}{\rightarrow} S B_{1, b-k} \rightarrow 1
$$

Using this and Lemma 4.13 we show that if elements of $K \cap S B_{a+k-1, b-k+1}$ are of the desired form, then so are elements of $K \cap S B_{a+k, b-k}$.

Let $X_{k}$ be an element of $K \cap S B_{a+k, b-k}$. By Lemma 4.13 we construct $W_{k} \in$ $K \cap S B_{a+k, b-k}$ such that $\operatorname{pr}^{\prime \prime}\left(W_{k}\right)=\operatorname{pr}^{\prime \prime}\left(X_{k}\right)$ and $W_{k}$ is a product of the desired elements. Using the above exact sequence $W_{k}^{-1} \cdot X_{k} \in K \cap S B_{a+k-1, b-k+1}$. If $W_{k}^{-1} \cdot X_{k}$ and $W_{k}$ are both of the desired form, then so is $X_{k}$.

Therefore by induction elements of $K \cap S B_{a+b}$ are of the desired form.

Corollary 4.15 Let $\lambda=\left(k_{1}^{a}, k_{2}^{b_{2}}, \ldots, k_{n}^{b_{m}}\right)$ with exponents satisfying $\sum_{2}^{m} b_{k}=b$ and $a \geq(3+\sqrt{9+8(2 g+b-2)}) / 2$. Additionally assume that for $2 \leq k \leq m$, we have $b_{k} \geq\left(3+\sqrt{9+8\left(2 g+b_{k+1}+\cdots+b_{m}-2\right)}\right) / 2$. Then any $Z \in \operatorname{ker}\left(\pi_{1}\left(\operatorname{Sym}_{g}^{\lambda}\right) \rightarrow\right.$ $\left.H_{1}(M, \mathbb{Z})\right)$ may be written as a product of $\sigma_{i j}, \kappa_{i j}$, and null $\rho_{r}, 1 \leq i, j \leq a+b$, $1 \leq r \leq 2 g$. 
Proof This differs from Theorem 4.14 only in that we eliminate the $i$-commutators, $a<i \leq a+b$. We may do this because any $i$-commutator is contained in the kernel of $\pi_{1}\left(\operatorname{Sym}^{b_{i}}\left(M_{b_{i+1}+\cdots+b_{m}}\right)\right) \rightarrow H_{1}(M, \mathbb{Z})$ and may thus by Corollary 4.6 be written as products of transpositions.

This gives us a description of $\operatorname{ker}\left(\mathrm{AJ}_{*}: \operatorname{Sym}_{g}^{\lambda} \rightarrow H_{1}(M, \mathbb{Z})\right)$ for $\lambda$ with at least one reasonably large set of points of the same weight (on the order of $\sqrt{g}$ ). The rest of the paper will be spent in determining which of the elements of this kernel are also elements of $\pi_{1}\left(\mathcal{Q}_{\lambda}\right)$. To do so we first present some methods for surgering existing quadratic differentials to create new ones.

\section{Some local surgeries}

We denote by $\mathcal{Q}_{\lambda}^{0}$ a connected component of $\mathcal{Q}_{\lambda}$, and by $\operatorname{cl}\left(\mathcal{Q}_{\lambda}\right)$ the closure of $\mathcal{Q}_{\lambda}$. Choose $\lambda_{1}, \lambda_{2}$ such that $\mathcal{Q}_{\lambda_{2}}^{0} \subset \operatorname{cl}\left(\mathcal{Q}_{\lambda_{1}}^{0}\right)$. In this section we present a method whereby, for certain $(M, q) \in \mathcal{Q}_{\lambda_{1}}^{0}$ sufficiently near to an element of $\mathcal{Q}_{\lambda_{2}}^{0}$, we may construct some transpositions and square transpositions of zeroes of $q$ in $\pi_{1}\left(\mathcal{Q}_{\lambda_{1}}^{0},(M, q)\right)$. For $\lambda=\left(k_{1}, \ldots, k_{n}\right)$ we refer to the zeroes of $q$ as $p_{1}, \ldots, p_{n}$ with $p_{i}$ of order $k_{i}$, and we let $P:=\left\{p_{1}, \ldots, p_{n}\right\}$. We also sometimes refer to a (square) transposition of $p_{i}$ and $p_{j}$ - by this we will mean a transposition if $k_{i}=k_{j}$ and a square transposition otherwise.

First we consider the following definition and two lemmas, which closely follow lemmas in [3] and [8], amongst others. The lemmas allow us in certain cases to construct an element of $\mathcal{Q}_{\lambda_{1}}^{0}$ from an element of $\mathcal{Q}_{\lambda_{2}}^{0}$. For a fixed $(M, q)$ and $p \in M$ let $D_{\varepsilon}(p)$ denote the disk of radius $\varepsilon$ centered at $p$ in the $q$ metric.

Definition 5.1 A zero $p$ of $(M, q)$ of order $k_{1}$ can be broken into zeroes of order $l_{1}, \ldots, l_{m}, \sum_{1}^{m} l_{i}=k_{1}$, if there exists $\varepsilon_{0}$ such that for every $\varepsilon<\varepsilon_{0}$ there exists a quadratic differential $\left(M^{\prime}, q^{\prime}\right)$ and a disk $D^{\prime} \subset M^{\prime}$ of radius $\varepsilon$ in the $q^{\prime}$ metric such that $M \backslash D_{\varepsilon}(p)$ is isometric to $M^{\prime} \backslash D^{\prime}$ and $D^{\prime}$ contains zeroes of order $l_{1}, \ldots, l_{m}$. If $(M, q) \in \mathcal{Q}_{g}\left(k_{1}, k_{2}, \ldots, k_{n}\right)$, then $\left(M^{\prime}, q^{\prime}\right) \in \mathcal{Q}_{g}\left(l_{1}, \ldots, l_{m}, k_{2}, \ldots, k_{n}\right)$.

Lemma 5.2 Let $(M, q) \in \mathcal{Q}_{g}\left(k_{1}, \ldots, k_{n}\right)$. Pick $l_{1}, l_{2} \in \mathbb{N}$ such that $l_{1}+l_{2}=k_{i}$, where if $k_{i}$ is even then $l_{1}$ and $l_{2}$ are even as well. Let $p_{i}$ be a zero of $q$ of order $k_{i}$. Then $p_{i}$ can be broken into zeroes of order $l_{1}, l_{2}$.

Lemma 5.3 Let $(M, q) \in \mathcal{Q}_{g}\left(k_{1}, \ldots, k_{n}\right)$. Pick $l_{1}, l_{2}, l_{3} \in \mathbb{N}$ such that $l_{1}+l_{2}+$ $l_{3}=k_{i}$. Then $p_{i}$ can be broken into zeroes of order $l_{1}, l_{2}, l_{3}$. Similarly, given any $l_{1}, l_{2}, l_{3}, l_{4} \in \mathbb{N}$ such that $l_{1}+l_{2}+l_{3}+l_{4}=k_{i}, p_{i}$ can be broken into zeroes of order $l_{1}, l_{2}, l_{3}, l_{4}$. 
Sketch of Proof A proof of all but the 4 point case may be found in [8]; we prove the two point case with both zeroes even here. Consider a disk $D_{\varepsilon}$, centered at $p_{i}$, of radius $\varepsilon$ in the flat metric given by $q$, where $\varepsilon$ is small enough that $D_{\varepsilon}$ contains no other zeroes of $q$. Since $p_{i}$ is of order $k_{i}, k_{i}+2$ horizontal trajectories will dead-end into $p_{i}$. Cut along these to make $k_{i}+2$ half-disks, each with a marked point given by $p_{i}$ half way along the cut. Pick some $\delta$ with $0<\delta<\varepsilon$. Construct two special half-disks by splitting the marked point given by $p_{i}$ into two marked points $2 \delta$ apart. Also shift the marked point given by $p_{i}$ by $\delta$ on the remaining disks, as in Figure 1. The trajectory structures on the individual half-disks do not change, and the half-disks

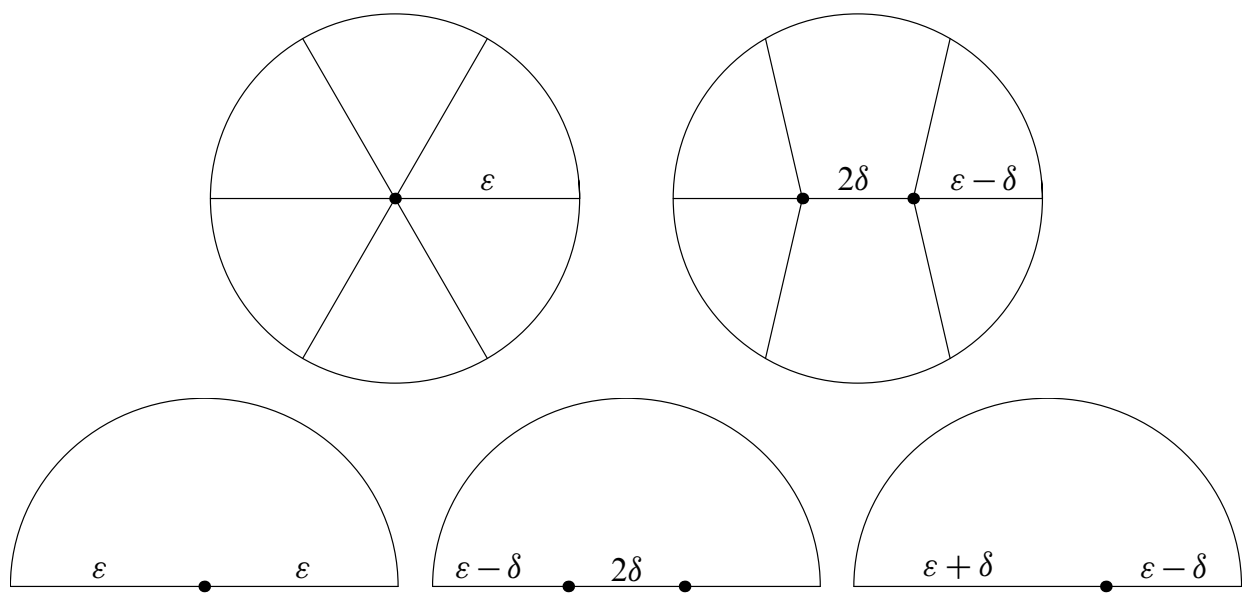

Figure 1: Taking a zero of order 4 to two zeroes of order 2 via splitting/moving the zero on individual half-disks. These diagrams are purely schematic and not to scale; the angle between any two straight lines in the disks is $\pi$.

can be glued back together, again as in Figure 1, to give an $\varepsilon$ disk containing zeroes of order $l_{1}, l_{2}$, with the same trajectory structure at the boundary as the original $D_{\varepsilon}$. We have a choice of the direction of the newly formed saddle connection: if we label one of the $k_{i}+2$ horizontal trajectories that dead-end into $p_{i}$ as $0^{\circ}$, then the new saddle connection can be in any direction $\theta, \theta \in\left[0,\left(k_{i}+2\right) \pi\right)$. The surgered surface is completely determined by $\delta$ and $\theta$. If $l_{1}=l_{2}=k_{i} / 2$ then the surface given by $\delta, \theta$ is the same as the surface given by $\delta, \theta+\left(l_{1}+1\right) \pi$, so we restrict ourselves to $\theta \in\left[0,\left(l_{1}+1\right) \pi\right)$.

The remaining two and three point cases are proved similarly to the one above. For the case of four zeroes, if any of the $l_{j}$ are even then we may apply so combination of two and three point surgeries to get the desired result. If $l_{1}, l_{2}, l_{3}, l_{4}$ are all odd then we 
cut $D_{\varepsilon}$ around $p_{i}$ into $k_{i}+2$ half-disks and glue as in Figure 2. Again, in all of these surgeries we have a choice of both $\delta$ and $\theta \in\left[0,\left(k_{i}+2\right) \pi\right)$.

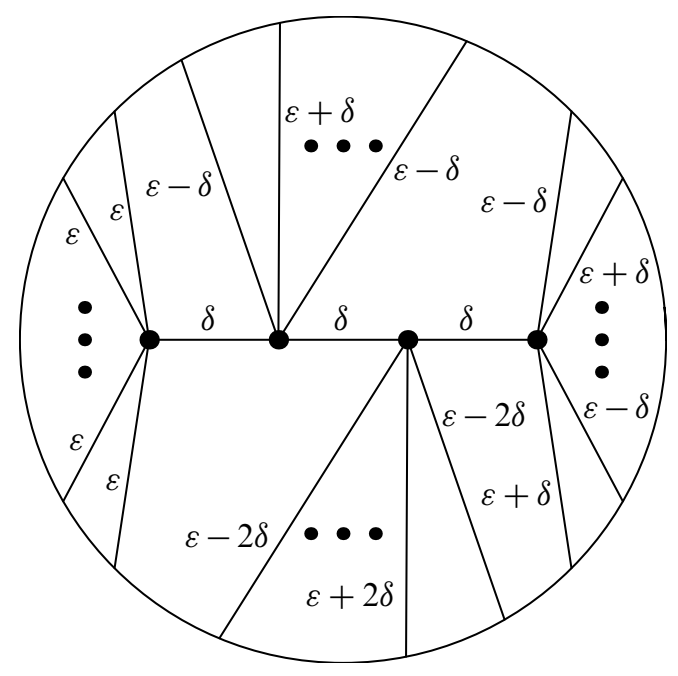

Figure 2: Splitting an even zero of high order into 4 odd zeroes of lower order

Define $\mathcal{Q}_{\lambda_{2}}^{0}$ to be a degeneration of $\mathcal{Q}_{\lambda_{1}}^{0}$ if it is possible to obtain an element in $\mathcal{Q}_{\lambda_{1}}^{0}$ by (possibly repeatedly) applying the surgeries of Lemma 5.2 and Lemma 5.3 to an element in $\mathcal{Q}_{\lambda_{2}}^{0}$. This puts a partial ordering on $\Lambda$, the set of partitions of $4 g-4$ : we say $\lambda_{1}>\lambda_{2}$ if there exists a component of $\mathcal{Q}_{\lambda_{2}}$ that is a degeneration of a component of $\mathcal{Q}_{\lambda_{1}}$. Note that if $\mathcal{Q}_{\lambda_{2}}^{0}$ is a degeneration of $\mathcal{Q}_{\lambda_{1}}^{0}$, then $\mathcal{Q}_{\lambda_{2}}^{0} \subset \operatorname{cl}\left(\mathcal{Q}_{\lambda_{1}}^{0}\right)$, but the converse is not necessarily true. For example, $\mathcal{Q}_{g}\left(1^{4 g-6}, 2\right)$ is in the closure of $\mathcal{Q}_{g}\left(1^{4 g-4}\right)$ $\left(\operatorname{cl}\left(\mathcal{Q}_{g}\left(1^{4 g-4}\right)=\mathcal{Q}_{g}\right.\right.$, so every stratum is in its closure), but $\mathcal{Q}_{g}\left(1^{4 g-6}, 2\right)$ is not a degeneration of $\mathcal{Q}_{g}\left(1^{4 g-4}\right)$.

Lemma 5.2 and Lemma 5.3 detail surgeries that allow us to break up zeroes of quadratic differentials; however, again following [3] we may collapse zeroes in the reverse process. We say three points, $p_{1}, p_{2}$, and $p_{3}$, are colinear if for a fixed $\theta$ there exist $\theta$-trajectories between $p_{1}, p_{2}$ and $p_{2}, p_{3}$.

Lemma 5.4 Suppose $\left(M^{\prime}, q^{\prime}\right) \in \mathcal{Q}_{g}\left(k_{1}, \ldots, k_{n}\right)$ is such that at least one of $k_{1}$ and $k_{2}$ is even, there exists a saddle connection between $p_{1}$ and $p_{2}$ of length $\delta$, and all other saddle connections from $p_{1}$ or $p_{2}$ are of length greater than $\delta$ for both even or $(3 / 2) \delta$ for one odd. Then there exists $(M, q) \in \mathcal{Q}_{g}\left(k_{1}+k_{2}, k_{3}, \ldots, k_{n}\right)$ such that $\left(M^{\prime}, q^{\prime}\right)$ may be obtained from $(M, q)$ via the surgery of Lemma 5.2. 
Suppose $\left(M^{\prime}, q^{\prime}\right) \in \mathcal{Q}_{g}\left(k_{1}, \ldots, k_{n}\right)$ is such that there exist saddle connections between $p_{1}, p_{2}$ and $p_{2}, p_{3}$ of length $\delta, p_{1}, p_{2}$, and $p_{3}$ are colinear, and all saddle connections from $p_{2}$ are of length at least $3 \delta$. Then there exists $(M, q) \in$ $\mathcal{Q}_{g}\left(k_{1}+k_{2}+k_{3}, k_{4}, \ldots, k_{n}\right)$ such that $\left(M^{\prime}, q^{\prime}\right)$ may be obtained from $(M, q)$ via the surgery of Lemma 5.2.

Proof We first prove the two point case. Label the points in each of the two top left disks of Figure 3 by $p_{1}$ and $p_{2}$. The top left disc (representing the one odd case) is contained in $D_{\delta / 2+\varepsilon}\left(p_{1}\right) \cup D_{\delta / 2+\varepsilon}\left(p_{2}\right)$. Then there exists $\varepsilon>\delta$ such that the disc contains no other zeroes of $q^{\prime}$. Since $\varepsilon>\delta$ we may reverse the cutting and pasting process of Lemma 5.2 to obtain $(M, q)$. In the left of the second pair of discs, the disc is again contained in $D_{\delta / 2+\varepsilon}\left(p_{1}\right) \cup D_{\delta / 2+\varepsilon}\left(p_{2}\right)$. Then there exists $\varepsilon>\delta / 2$ such that the disc contains no other zeroes of $q^{\prime}$, and since $\varepsilon>\delta / 2$ we may reverse the cutting and pasting process of Lemma 5.2 to obtain $(M, q)$.

For the three point case, label the points in the two bottom left discs of Figure 3 by $p_{1}, p_{2}, p_{3}$, with $p_{2}$ in the middle. $D_{\delta+\varepsilon}\left(p_{2}\right)$ contains either of these two discs. There exists $\varepsilon>2 \delta$ such that these disks contain no other zeroes of $q^{\prime}$ and we may reverse the cutting and pasting of Lemma 5.3 to obtain $(M, q)$.

In the next proposition we construct (square) transpositions of zeroes of $\left(M^{\prime}, q^{\prime}\right) \in \mathcal{Q}_{\lambda_{1}}$ by colliding two zeroes to get $(M, q)$ in a degeneration of $\mathcal{Q}_{\lambda_{1}}$, and then breaking up the newly formed zero of $q$ with respect to varying $\theta$. Breaking up a single zero into two zeroes in this manner gives us a transposition; however, breaking a single zero into three gives us something slightly different. Define a transposition of $p_{i}$ and $p_{j}$ $\bmod p_{k}$ to be an element of $\pi_{1}\left(\mathcal{Q}_{\lambda},(M, q)\right)$ such that $p_{i}, p_{j}$ follow paths that are not homotopic on $M \backslash P$, but are homotopic on $(M \backslash P) \cup p_{k}$. If $e$ is a path between $p_{i}, p_{j}$ through $p_{k}$ we refer to a transposition of $p_{i}, p_{j} \bmod p_{k}$ along $e$; by this we mean that $p_{i}$ and $p_{j}$ follow small deformations of $e$ on opposite sides of $p_{k}$. Similarly if we refer to a transposition of $p_{i}, p_{j}$ along $e$ we mean their transposition along a slight deformation of $e$ to one side of $p_{k}$.

Proposition 5.5 Let $\lambda_{1}=\left(k_{1}, k_{2}, \ldots, k_{n}\right), \lambda_{2}=\left(k_{1}+k_{2}, k_{3}, \ldots, k_{n}\right)$, and suppose $\left(M^{\prime}, q^{\prime}\right) \in \mathcal{Q}_{\lambda_{1}}$ satisfies the conditions of Lemma 5.4 in the two point case. Then there exists a trajectory of length $\delta$ between $p_{1}$ and $p_{2}$ (specified by Lemma 5.4), and an element of $\pi_{1}\left(Q_{\lambda_{1}},\left(M^{\prime}, q^{\prime}\right)\right)$ corresponding to the (square) transposition of $p_{1}, p_{2}$ along the specified trajectory.

Suppose $\lambda_{1}=\left(k_{1}, k_{2}, \ldots, k_{n}\right), \lambda_{3}=\left(k_{1}+k_{2}+k_{3}, k_{4}, \ldots, k_{n}\right)$, and $\left(M^{\prime}, q^{\prime}\right) \in$ $\mathcal{Q}_{\lambda_{1}}$ satisfies the conditions of Lemma 5.4 in the three point case. Then there exists a transposition of $p_{1}, p_{2} \bmod p_{3}$ along the union of two trajectories specified by Lemma 5.4. 


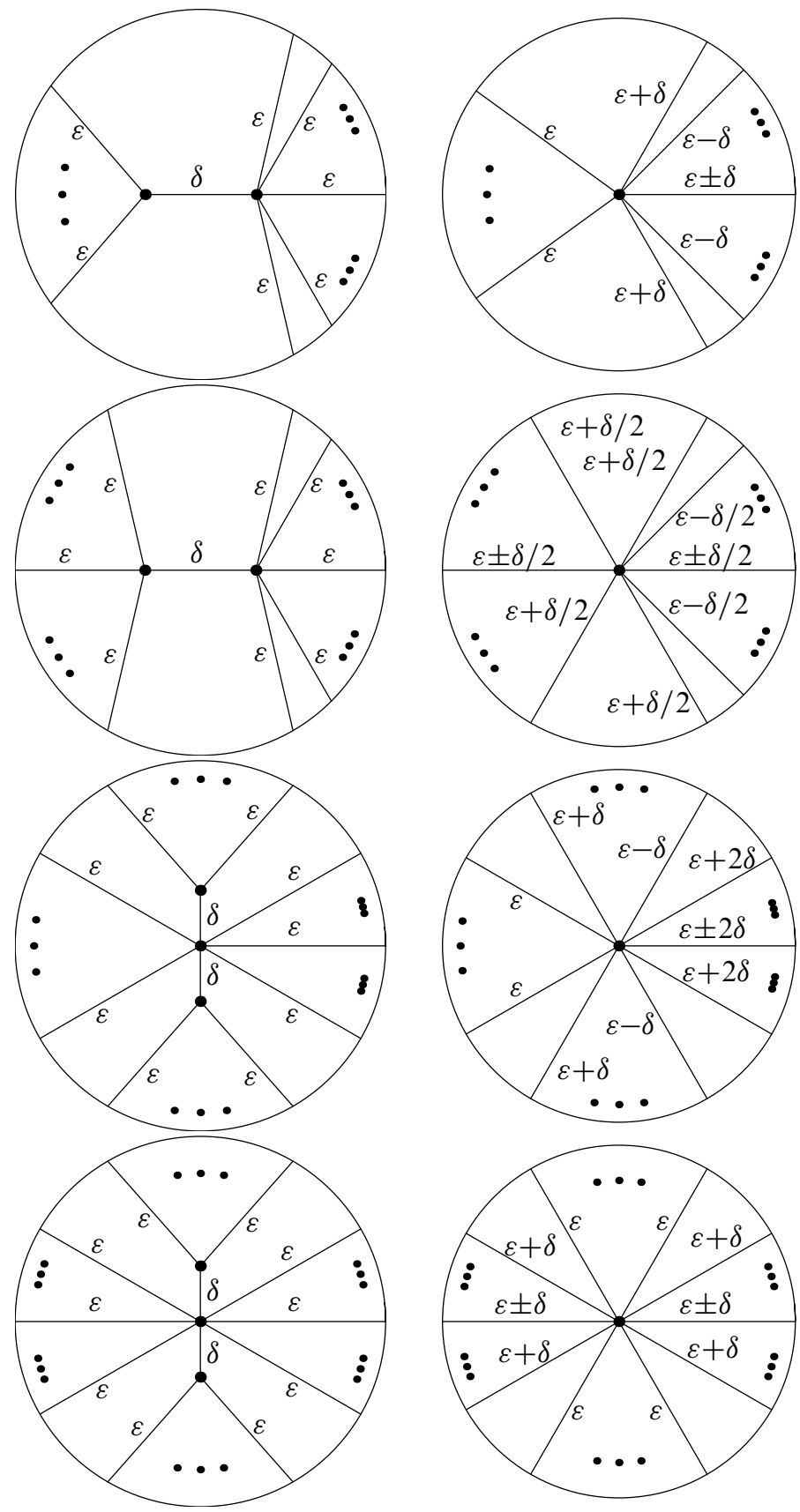

Figure 3: (i) Colliding two zeroes, one of odd order and one of even order. (ii) Colliding two zeroes of even order. (iii) Colliding three zeroes of odd order. (iv) Colliding three zeroes, two of any order and one even. 
Proof We first prove the proposition for the two point case. Suppose $\left(M^{\prime}, q^{\prime}\right)$ is obtained from $(M, q) \in \mathcal{Q}_{\lambda_{2}}$ by surgering with respect to $\delta_{0}, \theta_{0}$. Let $e_{0}$ be the new trajectory between $p_{1}, p_{2}$ of length $\delta_{0}$. Create a curve $\eta:\left[0, \delta_{0}\right] \rightarrow \operatorname{Cl}\left(\mathcal{Q}_{\lambda_{1}}\right)$ by surgering $(M, q)$ with respect to $t, \theta_{0}, t \in\left(0, \delta_{0}\right]$. Now, there exists a ball of some radius $\alpha$ around the zero of $q$ of order $k_{1}+k_{2}$ containing no other zeroes of $q$, so we apply the surgery of Lemma 5.2 to $(M, q)$ with respect to some fixed $\delta_{1}<\min \left\{\delta_{0}, \alpha\right\}$ and vary $\theta$ between 0 and either $\left(k_{1}+1\right) \pi$ or $\left(k_{1}+k_{2}+2\right) \pi$ (depending on whether we transpose or square transpose $p_{1}$ and $p_{2}$ ) to get a loop of surfaces, $\eta^{\prime} \subset \mathcal{Q}_{\lambda_{1}}$, centered at $(M, q) . \eta$ intersects $\eta^{\prime}$ at $\eta\left(\delta_{1}\right)$, and we define $\tilde{\eta}$ to be the subcurve of $\eta$ from $\delta_{0}$ to $\delta_{1}$. Then $\tilde{\eta} \circ \eta^{\prime} \circ \tilde{\eta}^{-1}$ gives us the desired loop in $\pi_{1}\left(\mathcal{Q}_{\lambda_{1}},\left(M^{\prime}, q^{\prime}\right)\right)$.

Following the same procedure for three points, we get a (square) transposition of $p_{1}, p_{2}$ mod $p_{3}$ along the union of the two trajectories between them in $\pi_{1}\left(Q_{\lambda_{1}},\left(M^{\prime}, q^{\prime}\right)\right)$.

Proposition 5.5 allows us to create (square) transpositions of various zeroes of a quadratic differential (possibly mod a third zero); however, the (square) transpositions created this way may be based at different elements of $\mathcal{Q}_{\lambda}$. In the next section we show that one way of obtaining elements based at the same $(M, q)$ is to consider hyperelliptic quadratic differentials.

\section{6 (Square) transpositions in certain strata}

In the next two sections we show that there exists a family of $\lambda$ for which $i_{*}\left(\pi_{1}\left(\mathcal{Q}_{\lambda}\right)\right)=$ $\operatorname{ker}\left(\mathrm{AJ}_{*}\right)$. We do so by explicitly constructing loops of quadratic differentials corresponding to the generators of $\operatorname{ker}\left(\mathrm{AJ}_{*}\right)$; in this section we construct the necessary (square) transpositions, and in the next section the null $\rho_{r}$ and $i$-commutators. For simplicity of notation we refer to the elements we construct as being in $\pi_{1}\left(\mathcal{Q}_{\lambda}\right)$.

We say that $\pi_{1}\left(\mathcal{Q}_{\lambda}^{0},(M, q)\right)$ contains all transpositions between $p_{i}, p_{j}$ if for all edges $e \in E_{M, q}$ between $p_{i}$ and $p_{j}, \sigma_{e}$ or $\kappa_{e} \in i_{*}\left(\pi_{1}\left(\mathcal{Q}_{\lambda}^{0},(M, q)\right)\right.$ ) (depending on whether $p_{i}$ and $p_{j}$ are of the same weight or not). We say that $\pi_{1}\left(\mathcal{Q}_{\lambda}^{0},(M, q)\right)$ contains all transpositions if the above is true for all pairs of zeroes of $q$. In Lemmas 6.1-6.4 we construct a variety of transpositions in hyperelliptic strata, and in the final proposition of the section we show that for some $\lambda$ the transpositions constructed in the four lemmas suffice to generate all transpositions in $\pi_{1}\left(\mathcal{Q}_{\lambda}^{0},(M, q)\right)$.

For any hyperelliptic quadratic differential, $(M, q)$, we let $\tau$ denote the hyperelliptic involution on $M$ and $\pi$ the degree 2 projection of $M$ to $\mathbb{P}^{1}$. We will use $\pi(M, q)$ to denote the projection of $q$ to $\mathbb{P}^{1}$. Notice that any zero of a hyperelliptic quadratic differential at a branch point of $\pi$ must be of even order. Unless specified otherwise we do not assume that the $k_{1}, \ldots, k_{n}$ in $\mathcal{Q}_{g}\left(k_{1}^{n_{1}}, k_{2}^{n_{2}}, \ldots, k_{m}^{n_{m}}\right)$ are distinct. 
Lemma 6.1 Let $\left(M_{0}, q_{0}\right) \in \mathcal{Q}_{\lambda}^{0}=\mathcal{Q}_{g}^{0}\left(k_{1}^{2}, \ldots, k_{m}^{2}, k_{m+1}, \ldots, k_{n}\right)$ be a hyperelliptic quadratic differential, and label the zeroes of $q_{0}$ by $p_{1}^{ \pm}, \ldots, p_{m}^{ \pm}, p_{m+1}, \ldots, p_{n}$. Suppose exactly $p_{m+1}, \ldots, p_{n}$ are at branch points of $M_{0}$ and at least one of $k_{1}, \ldots, k_{m}$, say $k_{l}$, is even. Let $e$ be an edge between $p_{i}^{+}, p_{j}^{+}, 1 \leq i<j \leq m$, such that $e \cap \tau(e)=\varnothing$. Then $\pi_{1}\left(\mathcal{Q}_{\lambda}^{0},\left(M_{0}, q_{0}\right)\right)$ contains an element corresponding to the (square) transposition of $p_{i}^{+}, p_{j}^{+}$along $e$. Analogous statements are true for $p_{i}^{+}, p_{j}^{-}$, and $p_{i}^{-}, p_{j}^{-}$.

Sketch of proof To prove the lemma we construct paths in the hyperelliptic locus of $\mathcal{Q}_{\lambda}$, from $\left(M_{0}, q_{0}\right)$ to quadratic differentials with discs that satisfy the conditions of Lemma 5.4 (as in Figure 3), and we then apply Proposition 5.5. In the case where at least one of $k_{i}, k_{j}$ is even, this proves the lemma. In the case where both $k_{i}$ and $k_{j}$ are odd the path we create moves $p_{i}, p_{j}$ near an even zero, $p_{l}$, and we create a (square) transposition of $p_{i}, p_{j} \bmod p_{l}$. However, since $k_{l}$ is even we also have a square transposition of $p_{j}$ and $p_{l}$, and the composition of the two gives us the desired (square) transposition of $p_{i}$ and $p_{j}$ (see Figure 4).

Proof Let $e:[0,1] \hookrightarrow M_{0}, e(0)=p_{i}^{+}, e(1)=p_{j}^{+}$, and let $\tilde{e}=\pi \circ e:[0,1] \rightarrow \mathbb{P}^{1}$ be the projection of $e$ to $\mathbb{P}^{1}$. By abuse of notation we also use $e, \tilde{e}$ to mean the images of $[0,1]$ under the maps $e$ and $\tilde{e}$.

We first assume at least one of $k_{i}, k_{j}$ is even. Define

$$
\begin{aligned}
\left(\mathbb{P}^{1}, \tilde{q}_{0}\right) & =\pi\left(M_{0}, q_{0}\right) \in \mathcal{Q}_{\tilde{\lambda}} \\
& =\mathcal{Q}_{0}\left(k_{1}, \ldots, k_{m}, \frac{k_{m+1}-2}{2}, \ldots, \frac{k_{n}-2}{2},-1^{2 g+2-(n-m)}\right)
\end{aligned}
$$

and label the singularities of $\tilde{q}_{0}$ by $\tilde{p}_{1}, \tilde{p}_{2}, \ldots, \tilde{p}_{2 g+2+m}$, such that $\pi\left(p_{s}^{ \pm}\right)=\tilde{p}_{s}$ for $1 \leq s \leq m$ and $\pi\left(p_{t}\right)=\tilde{p}_{t}$ for $m<t \leq n$. By Proposition 2.2 we may construct a quadratic differential on $\mathbb{P}^{1}$ with arbitrary zeroes; thus, define $\left(\mathbb{P}^{1}, \tilde{q}_{t}\right) \in \mathcal{Q}_{\tilde{\lambda}}$ to be the quadratic differential with zeroes at $\tilde{p}_{1}, \ldots, \tilde{p}_{i-1}, \widetilde{e}(t), \tilde{p}_{i+1}, \ldots, \tilde{p}_{2 g+2+m}$. Let $\left(M_{t}, q_{t}\right) \in \mathcal{Q}_{\lambda}^{0}$ be the double cover of $\left(\mathbb{P}^{1}, \tilde{q}_{t}\right)$, ramified at $\tilde{p}_{m+1}, \ldots, \widetilde{p}_{2 g+2+m}$.

As $t$ approaches $1, e(t)$ approaches $p_{j}^{+}$, and there exists $t_{0}$ close to 1 such that $\left(M_{t_{0}}, q_{t_{0}}\right)$ satisfies the conditions of Lemma 5.4. Then we may apply Proposition 5.5 to obtain a (square) transposition, $T \in \pi_{1}\left(Q_{\lambda}^{0},\left(M_{t_{0}}, q_{t_{0}}\right)\right)$, of $p_{j}$ and $e\left(t_{0}\right)$ along a trajectory homotopic to $e\left(\left[t_{0}, 1\right]\right)$. Let $E$ be the image of $\left[0, t_{0}\right]$ in $\mathcal{Q}_{\lambda}^{0}$ under $t \mapsto$ $\left(M_{t}, q_{t}\right)$. Along $E^{-1} \circ T \circ E p_{i}^{+}$and $p_{j}^{+}$(square) transpose along $e$. Further, $p_{i}^{-}$ follows a trivial path (since $e \cap \tau(e)=\varnothing$ ) and all other zeroes stay constant, so this gives us the desired (square) transposition. 
Now suppose both $k_{i}$ and $k_{j}$ are odd. Let $e$ be as above and recall that $k_{l}$ is even. let $\tilde{e}^{\prime}:[0,1] \hookrightarrow \mathbb{P}^{1}$ be an edge from $\tilde{p}_{l}$ to $\tilde{p}_{j}$ such that $\tilde{e}^{\prime} \cap \tilde{e}=\tilde{p}_{j}$, and let $e^{\prime}:[0,1] \hookrightarrow M_{0}$ be the edge between $p_{j}^{+}$and either $p_{l}^{+}$or $p_{l}^{-}$that projects to $\widetilde{e}^{\prime}$. Since $\widetilde{e}^{\prime}$ does not intersect itself $e^{\prime} \cap \tau\left(e^{\prime}\right)=\varnothing$, and also by construction $e^{\prime} \cap e=p_{j}^{+}$.

Define $\left(\mathbb{P}^{1}, \tilde{q}_{t}^{\prime}\right) \in \mathcal{Q}_{\tilde{\lambda}}$ to be the quadratic differential with zeroes at $\tilde{p}_{1}, \ldots, \tilde{p}_{i-1}$, $\widetilde{e}(t), \tilde{p}_{i+1}, \ldots, \tilde{p}_{l-1}, \widetilde{e}^{\prime}(t), \tilde{p}_{l+1}, \ldots, \tilde{p}_{2 g+2+m}$, and let $\left(M_{t}^{\prime}, q_{t}^{\prime}\right) \in \mathcal{Q}_{\lambda}^{0}$ be the double cover of $\left(\mathbb{P}^{1}, \tilde{q}_{t}^{\prime}\right)$, ramified at $\tilde{p}_{m+1}, \ldots, \widetilde{p}_{2 g+2+m}$. Again there will be some $t_{0}$ such that $\left(M_{t_{0}}^{\prime}, q_{t_{0}}^{\prime}\right)$ satisfies the conditions of Lemma 5.4. (Some slight deformation of $e$ and $e^{\prime}$ may be required to get $e(t), e^{\prime}(t)$ and $p_{j}^{+}$colinear). Then by Proposition 5.5, $\pi_{1}\left(\mathcal{Q}_{\lambda},\left(M_{t_{0}}^{\prime}, q_{t_{0}}^{\prime}\right)\right)$ contains a transposition of $e\left(t_{0}\right)$ and $p_{j} \bmod e^{\prime}\left(t_{0}\right)$ along $e\left(\left[t_{0}, 1\right]\right)$. However, since $e^{\prime}\left(t_{0}\right)$ is a zero of even order there exists a square transposition of $e^{\prime}\left(t_{0}\right)$ and $p_{j}$ along $e^{\prime}\left(\left[t_{0}, 1\right]\right)$, also in $\pi_{1}\left(\mathcal{Q}_{\lambda},\left(M_{t_{0}}, q_{t_{0}}\right)\right)$. The composition of this square transposition and the transposition of $e\left(t_{0}\right)$ and $p_{j} \bmod e^{\prime}\left(t_{0}\right)$ is shown in Figure 4 , and gives a true transposition of $e\left(t_{0}\right)$ and $p_{j}$ along $e\left(\left[t_{0}, 1\right]\right)$. The rest of the proof follows as in the even case.
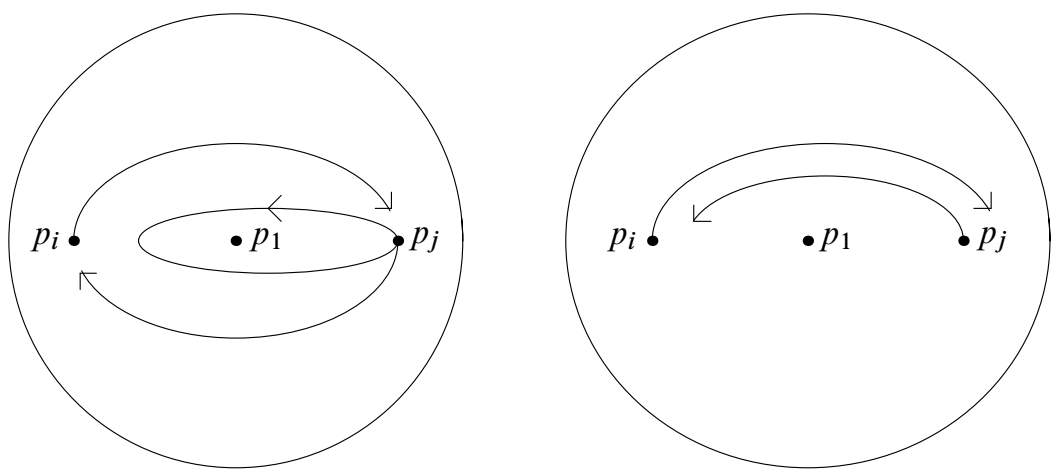

Figure 4: Homotopic paths along which to transpose $p_{i}, p_{j}$, of odd order when $p_{l}$ is of even order

Notice that any saddle connection, $e$, between two branch points of a hyperelliptic quadratic differential will have a "twin" saddle connection, $\tau(e)$, parallel and of the same length. We would like to prove a lemma similar to Lemma 6.1 for branch zeroes; however, colliding two branch zeroes along $e$ will also cause the length of $\tau(e)$ to go to zero and a homology cycle to collapse. We deal with this by leaving the hyperelliptic locus of $\mathcal{Q}_{\lambda}$ shortly before colliding the two zeroes, and showing that for a generic element outside the hyperelliptic locus, the saddle connections corresponding to $e$ and $\tau(e)$ will be of different lengths. 
In particular, for any $(M, q)$ pick $x \in M \backslash P$ and notice that for any loop, $\gamma$ on $M \backslash P$ based at $x$ and $v \in T_{x} M$, parallel transport along $\gamma$ takes a vector $v$ either to $v$ or $-v$. This gives us a homomorphism

$$
\text { hol: } \pi_{1}(M \backslash P, x) \rightarrow \mathbb{Z}_{2} \text {. }
$$

Following Masur and Zorich in [10], this gives us a double cover of $M, \widehat{M}$, endowed with an induced flat metric that by construction has trivial holonomy and thus defines an abelian differential, $(\widehat{M}, \widehat{q}) \in \mathcal{H} \hat{\lambda}$ of genus $\widehat{g}$, with zeroes $\widehat{P} . \widehat{M}$ is the canonical double cover of $M$, and has a natural involution, $\varphi$. Let $e$ be an oriented saddle connection (either closed or not) on $M$, and let $\hat{e}^{\prime}, \hat{e}^{\prime \prime}$ be its lifts to $\widehat{M}$. The lifts $\hat{e}^{\prime}$ and $\widehat{e}^{\prime \prime}$ both determine homology classes $\left[\widehat{e}^{\prime}\right],\left[\hat{e}^{\prime \prime}\right] \in H_{1}(\widehat{M}, \widehat{P} ; \mathbb{Z})$. Again following [10], if $\left[\hat{e}^{\prime}\right]=-\left[\hat{e}^{\prime \prime}\right]$ we define $[\hat{e}]:=\left[\hat{e}^{\prime}\right]$; otherwise, we define $[\hat{e}]:=\left[\hat{e}^{\prime}\right]-\left[\hat{e}^{\prime \prime}\right]$. We then say two saddle connections, $e_{1}$ and $e_{2}$, are homologous if $\left[\hat{e}_{1}\right]= \pm\left[\widehat{e}_{2}\right]$ in $H_{1}(\widehat{M}, \widehat{P} ; \mathbb{Z})$. Now pick $\left(M_{0}, \omega\right) \in \mathcal{H} \hat{\lambda}$ with zeroes $P_{0}$, and let $\gamma \in H_{1}\left(M^{\prime}, P^{\prime} ; \mathbb{Z}\right)$. The period of $\gamma$ is $|\gamma|:=\int_{\gamma} \omega^{\prime}$. Fix a basis $\left(\gamma_{1}, \ldots, \gamma_{2} \hat{g}+n-1\right)$ of $H_{1}\left(M_{0}, P_{0} ; \mathbb{Z}\right)$. Any nearby element, $\left(M^{\prime}, \omega^{\prime}\right) \in \mathcal{H} \hat{\lambda}$ has the same underlying topological surface and singular points, and therefore a well-defined notion of $\left(\gamma_{1}, \ldots, \gamma_{2} \widehat{g}+n-1\right)$. Then we have a local map $\mathcal{H} \widehat{\lambda} \rightarrow H^{1}\left(M_{0}, P_{0} ; \mathbb{C}\right) \cong \mathbb{C}^{2 \widehat{g}+n-1}$,

$$
\left(M^{\prime}, \omega^{\prime}\right) \mapsto\left(\left|\gamma_{1}\right|, \ldots,\left|\gamma_{2} \widehat{g}+n-1\right|\right) .
$$

This is the called the period map and is a local homeomorphism of a neighborhood of $\left(M^{\prime}, \omega^{\prime}\right)$ with a domain of $\mathbb{C}^{2 \hat{g}+n-1}$. This gives us a local coordinates on $\mathcal{H} \hat{\lambda}$ and an induced Lebesgue measure on $\mathcal{H} \hat{\lambda}$. Back to $(\widehat{M}, \widehat{q})$, let $H^{1-}(\widehat{M}, \widehat{P} ; \mathbb{C})$ be the subspace of $H^{1}(\widehat{M}, \widehat{P} ; \mathbb{Z})$ that is anti-invariant with respect to $\varphi^{*}$. Notice that locally elements of $\mathcal{Q}_{\lambda}$ will have double covers in the same stratum of abelian differentials. abelian differentials whose images lie in $H^{1-}(\widehat{M}, \widehat{P} ; \mathbb{C})$ are precisely those that arise from quadratic differentials in $\mathcal{Q}_{\lambda}$. This gives a local homeomorphism between a neighborhood of $\mathcal{Q}_{\lambda}$ and a open domain of $\mathbb{C}^{2 g+n-2}$, and again a corresponding Lebesgue measure on $\mathcal{Q}_{\lambda}$. See Lanneau [8], for example. If $e_{1}$ and $e_{2}$ are not $\widehat{h}$ omologous, the homology classes of $\widehat{e}_{1}, \widehat{e}_{2}$ are independent in $H_{1}^{-}(\widehat{M}, \widehat{P}, \mathbb{Z})$, and $\left|\left[\hat{e}_{1}\right]\right|=\left|\left[\hat{e}_{2}\right]\right|$ only on a set of measure zero. Since $|[e]|:=\left|\int_{e} q\right|=\left|\int_{\widehat{e}^{\prime}} \hat{q}\right|=(1 / 2)\left|\int_{\widehat{e}} \hat{q}\right|$, the same will be true of $e_{1}$ and $e_{2}-$ if they are non- $\hat{h}$ omologous $\left|\left[e_{1}\right]\right|=\left|\left[e_{2}\right]\right|$ only on a set of measure zero.

Lemma 6.2 Let $\left(M_{0}, q_{0}\right)$ and $\lambda$ be as in Lemma 6.1, with some $k_{i}$ odd, $1 \leq i \leq n$, at least two branch zeroes, and $g>0$. Let $e^{+}$be a saddle connection between two of the branch zeroes of $q_{0}$, and let $e^{-}:=\tau\left(e^{+}\right)$. Then there exists some open set, $U \subset Q_{\lambda}$, containing $\left(M_{0}, q_{0}\right)$, such that for almost every element of $U$ these saddle connections are not of the same length. 
Proof As noted above, if $e^{+}$and $e^{-}$are not $\hat{h}$ omologous in $H_{1}^{-}\left(\widehat{M}_{0}, \widehat{P}_{0} ; \mathbb{Z}\right)$ then they are generically not of the same length. Thus, to prove the lemma we suppose that $[\widehat{e}]:=\left[\hat{e}^{+}\right]-\left[\hat{e}^{-}\right]=0$ and show a contradiction.

If $[\hat{e}]=0$ then $\hat{e} \in[\hat{e}]$ is a separating curve on $\widehat{M}_{0}$. Let $\widetilde{M}_{0}$ be the surface with boundary obtained by cutting $M_{0}$ along $e^{+} \cup e^{-}$, which together form a nontrivial cycle, and let $p \in \widetilde{M}_{0} \backslash P_{0}$. Since $g>0, \widetilde{M}_{0}$ is connected. If any element of $\pi_{1}\left(\widetilde{M}_{0} \backslash P_{0}, p\right)$ mapped to -1 under hol: $\pi_{1}\left(\widetilde{M}_{0} \backslash P_{0}, p\right) \rightarrow \mathbb{Z}_{2}$ then $\widehat{M}_{0}$ cut at $\widehat{e}$ would still be connected, which implies that every element of $\pi_{1}\left(\widetilde{M}_{0} \backslash P_{0}, p\right)$ maps to 1 under hol. But by assumption one of the zeroes of $P_{0}$ is odd and a small loop around it will map to -1 under hol, giving us the desired contradiction.

Now we can prove the analog of Lemma 6.1 for branch zeroes.

Lemma 6.3 Let $\left(M_{0}, q_{0}\right), \lambda$ and $\tilde{\lambda}$ be as in Lemma 6.1, with at least one $k_{i}$ odd, $1 \leq i \leq n$, and let $e$ be any edge between two branch zeroes of $q_{0}, p_{i}$ and $p_{j}$, $m<i<j \leq n$. Then $\pi_{1}\left(\mathcal{Q}_{\lambda}^{0},\left(M_{0}, q_{0}\right)\right)$ contains a (square) transposition of $p_{i}, p_{j}$ along $e$.

Proof Let $e:[0,1] \hookrightarrow M_{0}, e(0)=p_{i}, e(1)=p_{j}$, be the edge between $p_{i}, p_{j}$, and let $\tilde{e}:=\pi \circ e$ be its projection to $\mathbb{P}^{1}$. Let

$$
\tilde{\lambda}=\left(k_{1}, \ldots, k_{m}, \frac{k_{m+1}-2}{2}, \ldots, \frac{k_{n}-2}{2},-1^{2 g+2-(n-m)}\right) .
$$

Let $\left(\mathbb{P}^{1}, \widetilde{q}_{0}\right)=\pi\left(M_{0}, q_{0}\right) \in \mathcal{Q}_{\tilde{\lambda}}$ and label the singularities of $\widetilde{q}_{0}$ as $\tilde{p}_{1}, \tilde{p}_{2}, \ldots$, $\tilde{p}_{2 g+2+m}$. Define $\left(\mathbb{P}^{1}, \tilde{q}_{t}\right)$ to be the element of $\mathcal{Q}_{\tilde{\lambda}}$ with singularities at $\tilde{p}_{1}, \widetilde{p}_{2}, \ldots$, $\tilde{p}_{i-1}, \widetilde{e}(t), \tilde{p}_{i+1}, \ldots, \tilde{p}_{2 g+2+m}$. Taking a double cover of $\left(\mathbb{P}^{1}, \tilde{q}_{t}\right)$ branched at its last $2 g+2$ singularities we get an element of $\mathcal{Q}_{\lambda}$, which we call $\left(M_{t}, q_{t}\right)$.

As $t$ approaches $1, e(t)$ approaches $p_{j}$ and all other $p_{k}$ are fixed. As in Lemma 6.1 we wish to show there exists a subset of some $M_{t}$ containing $e(t)$ and $p_{j}$, and satisfying the conditions of Lemma 5.4. In particular, if $e(t)$ and $p_{j}$ are $\delta$ apart it suffices to show that all other saddle connections on $M_{t}$ are of length greater than $\delta$. This condition is not initially satisfied because on $\left(M_{t}, q_{t}\right)$ there are two short saddle connections of the same length running between $e(t)$ and $p_{j}$. However, Lemma 6.2 implies that there exists $(M, q)$ arbitrarily close to any $\left(M_{t}, q_{t}\right)$ such that the saddle connection in the homotopy classes of $e([t, 1])$ and $\tau(e([t, 1]))$ are of different lengths. Because of the involution on $\left(M_{t}, q_{t}\right)$ we may assume the saddle connection on $(M, q)$ in the homotopy class of $e([t, 1])$ is shorter and thus $(M, q)$ satisfies the conditions of Lemma 5.4. Then there exists a (square) transposition of $e(t), p_{j}$ along $e([t, 1])$, based at $(M, q)$. The rest of the argument follows as in Lemma 6.1. 
Lemma 6.4 Let $\left(M_{0}, q_{0}\right)$ and $\lambda$ be as in Lemma 6.1, and let $b$ be a branch point of $M_{0}$ that is also a regular point of $q_{0}$. Let $e_{b}$ be an edge between $b$ and $p_{i}^{+}$, $1 \leq i \leq m$, such that $\tau\left(e_{b}\right) \cap e_{b}=b$. Then $e:=e_{b} \cup \tau\left(e_{b}\right)$ is an edge between $p_{i}^{+}$ and $p_{i}^{-}$, and $\pi_{1}\left(\mathcal{Q}_{\lambda}^{0},\left(M_{0}, q_{0}\right)\right)$ contains an element corresponding to the transposition of $p_{i}^{+}, p_{i}^{-}$along $e$.

Proof Let $e^{\prime}$ be a small deformation of $e$ to one side of $b$ that is still an edge. We can always choose $e^{\prime}$ so that $e^{\prime} \sim e \sim \tau\left(e^{\prime}\right)$ and $e^{\prime} \cap \tau\left(e^{\prime}\right)=p_{i}^{ \pm}$. As in the previous two lemmas, let $\left(\mathbb{P}^{1}, \tilde{q}_{0}\right)=\pi\left(M_{0}, q_{0}\right)$, with singularities $\tilde{p}_{1}, \ldots, \tilde{p}_{2 g+2+m}$, and let $\widetilde{e}^{\prime}=\pi\left(e^{\prime}\right)$. Define $\left(\mathbb{P}^{1}, \tilde{q}_{t}\right)$ to be the quadratic differential with zeroes at $\tilde{p}_{1}, \ldots, \tilde{p}_{i-1}, \tilde{e}^{\prime}(t), \tilde{p}_{i+1}, \ldots, \tilde{p}_{2 g+2+m}$, and let $\left(M_{t}, q_{t}\right)$ be its double cover ramified at $\tilde{p}_{m+1}, \ldots, \tilde{p}_{2 g+2+m}$. Then $\left(M_{0}, q_{0}\right)=\left(M_{1}, q_{1}\right)$ and $T:[0,1] \rightarrow \mathcal{Q}_{\lambda}, t \mapsto$ $\left(M_{t}, q_{t}\right)$ is the desired element of $\pi_{1}\left(\mathcal{Q}_{\lambda},\left(M_{0}, q_{0}\right)\right)$.

The previous four lemmas will allow us to show that certain hyperelliptic strata contain all of their transpositions, but first we need one more technical lemma. Recall that the maximal number of faces a planar graph with $n$ vertices can have is $2 n-4$. (Such a graph will be a triangulation, with $3 n-6$ edges.) By removing edges from such a graph it is always possible to construct a planar graph with $n$ vertices and fewer than $2 n-4$ faces. (As earlier in the paper, we do not allow graphs to have double edges or loops.)

Lemma 6.5 Let $\Gamma$ be a planar graph with $n$ vertices and $f$ faces, $f \leq 2 n-4$. Then we may associate to each face of $\Gamma$ a pair of vertices adjacent to the face such that the same pair is not associated to more than 1 face.

Proof It suffices to associate a unique adjacent edge to each face of $\Gamma$, since this is equivalent to associating the two vertices adjacent to the edge to the face. Pick any face, $F_{1}$, of $\Gamma$ and any edge, $e_{1}$, adjacent to $F_{1}$. The edge $e_{1}$ is adjacent to one other face, which we call $F_{2} . F_{2}$ has at least two other possible adjacent edges, so again pick any edge $e_{2} \neq e_{1}$ to associate to $F_{2}$, and let $F_{3}$ be the other face adjacent to $e_{2}$. Continue this process until one of two things happens. Either an edge is associated to each face, or at stage $k F_{k}=F_{i}$ for $1 \leq i \leq k$ and $k \neq f$. In the first case we are done, and in the second the remaining faces of the graph do not have any adjacent edges that have been associated to any other face, so we pick any face, call it $F_{k+1}$ and resume the process.

Proposition 6.6 Suppose that $\mathcal{Q}_{\lambda}=\mathcal{Q}_{g}\left(1^{2 n}, k_{1}, \ldots, k_{m}\right)$ is such that $2 n \geq g+5$, $k_{1}, \ldots, k_{m}$ are all even, and there exists $i, j, 1 \leq i<j \leq m$, such that $k_{i}=k_{j}$. Then for any $(M, q) \in \mathcal{Q}_{\lambda}, \pi_{1}\left(\mathcal{Q}_{\lambda},(M, q)\right)$ contains all transpositions. 
Sketch of Proof Our plan is to construct a hyperelliptic $(M, q)$ with higher order zeroes at branch points, and then put a graph on $M$ with the single zeroes of $q$ at the vertices and at most one higher order zero in each face. We use Lemma 6.1 and Lemma 6.4 to construct the transpositions associated to each edge of the graph, which via Theorem 4.2 gives us all (square) transpositions of single zeroes with each other and with higher order zeroes. We then apply Lemma 6.3 to get (square) transpositions of the higher order zeroes with each other.

Proof By Theorem $2.7 \mathcal{Q}_{\lambda}$ is connected so we need not consider connected components. Without loss of generality suppose $k_{1}=k_{2}$. Put a graph $\widetilde{\Gamma}$ on $\mathbb{P}^{1}$ with $n$ vertices and $g+1$ faces. Since $n \geq(g+5) / 2, g+1 \leq 2 n-4$, and it is always possible to construct such a graph. Mark 2 points in each of $g$ faces of $\widetilde{\Gamma}$, and 3 points in the $(g+1)$-st. By Proposition 2.2 there exists $\widetilde{q}$ on $\mathbb{P}^{1}$ such that the vertices of $\widetilde{\Gamma}$ are zeroes of $\widetilde{q}$ of order $1, m-1$ marked points are zeroes of $\widetilde{q}$ of order $k_{1},\left(k_{3}-2\right) / 2,\left(k_{4}-2\right) / 2, \ldots,\left(k_{m}-2\right) / 2$, and the $2 g+3-m$ remaining marked points are poles of $\tilde{q}$ of order 1 . Since $m<2 g+2, \tilde{q}$ has at least 2 poles and we assume the $(g+1)$-st face contains the zero of order $k_{1}$ and 2 poles. Denote the $n$ single zeroes of $\widetilde{q}$ by $p_{1}, \ldots, p_{n}$. To each face of $\widetilde{\Gamma}$ we associate a pair of vertices as in Lemma 6.5 .

Take a double cover of $\left(\mathbb{P}^{1}, \widetilde{q}\right)$ ramified at the $2 g+2$ marked points in the faces of $\widetilde{\Gamma}$, minus the zero of order $k_{1}$. Assume each branch cut is between two points in the same face and is contained in that face. This gives us $(M, q) \in \mathcal{Q}_{g}\left(1^{2 n}, k_{1}, k_{2}, \ldots, k_{m}\right)$. $(M, q)$ has two copies of $\widetilde{\Gamma}$ embedded into it, $\widetilde{\Gamma}^{+}$and $\widetilde{\Gamma}^{-}$; denote their vertices by $p_{1}^{+}, \ldots, p_{n}^{+}$and $p_{1}^{-}, \ldots, p_{n}^{-}$, with $\tau\left(p_{l}^{+}\right)=p_{l}^{-}, 1 \leq l \leq n$. By associating a pair of vertices to each face of $\widetilde{\Gamma}$ we have associated a quadruplet of vertices, $p_{i}^{ \pm}$and $p_{j}^{ \pm}$, to each of the $g+1$ branch cuts of $M$. Construct a pair of edges, $e_{k}$ and $\tau\left(e_{k}\right)$, between $p_{i}^{+}, p_{j}^{-}$and $p_{j}^{+}, p_{j}^{-}$through the $k$-th branch cut, $1 \leq k \leq g$, as in Figure 5 . Notice that we may always construct $e_{k}$ and $\tau\left(e_{k}\right)$ so that they do not intersect. For the $(g+1)$-st branch cut, both branch points are regular and we construct edges, $e_{g+1}$ and $e_{g+1}^{\prime}$ between $p_{i}^{ \pm}$and $p_{j}^{ \pm}$, as in Lemma 6.4. An example of this is shown in Figure 6. The union $\widetilde{\Gamma}^{+} \cup \widetilde{\Gamma}^{-} \cup e_{1} \cup \tau\left(e_{1}\right) \cup \cdots \cup e_{g} \cup \tau\left(e_{g}\right) \cup e_{g+1} \cup e_{g+1}^{\prime}$ gives us a connected graph $\Gamma$ on $M$ with $2 g+2$ faces and two faces associated to each branch cut. This graph will be a 2-cell embedding and by construction satisfies all of the hypotheses of Theorem 4.2. Exactly one branch point is in each of faces associated to the first $g$ branch cuts. For the two faces, $F$ and $F^{\prime}$, associated to the $(g+1)-$ st branch cut, $F^{\prime}=\tau(F)$ so again there is one zero of order $k_{1}\left(=k_{2}\right)$ in each of $F, F^{\prime}$.

By Lemma 6.1 we may construct an element of $\pi_{1}\left(\mathcal{Q}_{\lambda},(M, q)\right)$ corresponding to the transposition of $p_{i}^{+}, p_{j}^{-}$along both $e_{k}$ and $\tau\left(e_{k}\right)$ for $1 \leq k \leq g$. Further, each edge $e^{+}$ 

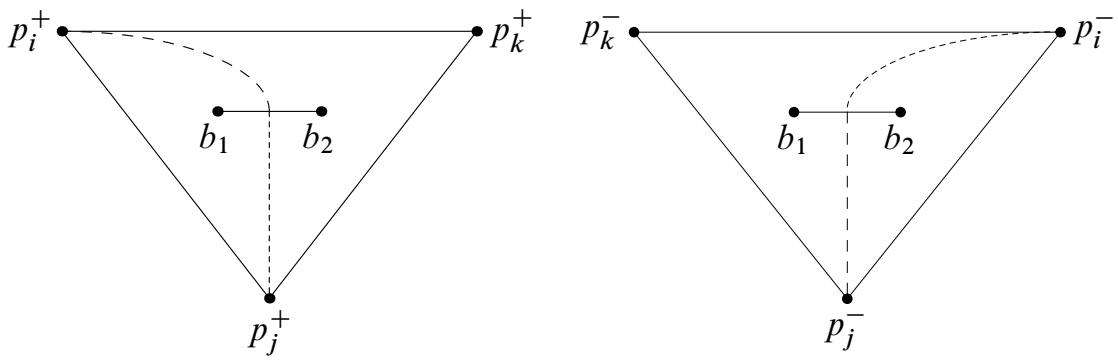

Figure 5: The edges $e_{k}$ and $\tau\left(e_{k}\right)$ between $p_{i}^{+}, p_{j}^{-}$and $p_{i}^{-}, p_{j}^{+} . b_{1}$ and $b_{2}$ denote branch points, and the line between them denotes a branch cut.
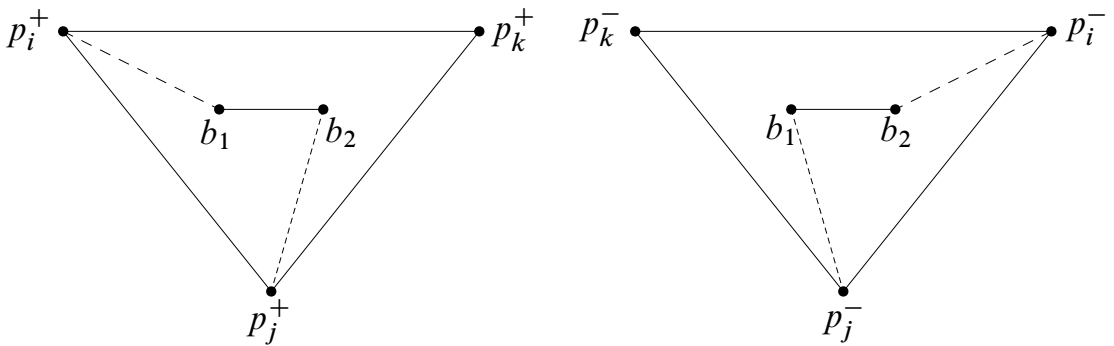

Figure 6: The edges $e_{g+1}$ and $e_{g+1}^{\prime}$ between $p_{i}^{+}, p_{i}^{-}$and $p_{j}^{-}, p_{j}^{-}$

of $\widetilde{\Gamma}^{+}$is contained entirely on one sheet of $M$, so $e^{+} \cap \tau\left(e^{+}\right)=\varnothing$ and again by Lemma $6.1 \sigma_{e^{+}}, \sigma_{e^{-}} \in \pi_{1}\left(\mathcal{Q}_{\lambda},(M, q)\right)$. Lemma 6.4 gives us transpositions associated to $e_{g+1}$ and $e_{g+1}^{\prime}$. Consequently for every edge $e$ of $\Gamma, \sigma_{e} \in \pi_{1}\left(\mathcal{Q}_{\lambda},(M, q)\right)$. Since there is at most one higher order zero in each face, Theorem 4.2 implies $\pi_{1}\left(\mathcal{Q}_{\lambda},(M, q)\right)$ contains all transposition of the $2 n$ zeroes of order 1 with each other, and all square transpositions of zeroes of order 1 with zeroes of order $k_{1}, \ldots, k_{m}$.

Now pick any $\left(\mathbb{P}^{1}, \widetilde{q}^{\prime}\right) \in \mathcal{Q}_{0}\left(1^{n},\left(k_{1}-2\right) / 2, \ldots,\left(k_{m}-2\right) / 2,-1^{2 g+2-m}\right)$, and let $\left(M^{\prime}, q^{\prime}\right) \in \mathcal{Q}_{\lambda}$ be its double cover, ramified at the zeroes of $\widetilde{q}^{\prime}$ not of order 1 . Then all of the even zeroes of $q^{\prime}$ are at branch points of $M^{\prime}$ and by Lemma $6.3 \pi_{1}\left(\mathcal{Q}_{\lambda},\left(M^{\prime}, q^{\prime}\right)\right)$ contains all (square) transpositions of zeroes of order $k_{1}, \ldots, k_{m}$ with each other. But $\pi_{1}\left(\mathcal{Q}_{\lambda},\left(M^{\prime}, q^{\prime}\right)\right)$ is isomorphic to $\pi_{1}\left(\mathcal{Q}_{\lambda},(M, q)\right)$ so the same is then true for $\pi_{1}\left(\mathcal{Q}_{\lambda},(M, q)\right)$. This proves the proposition.

\section{Constructing the remaining generators}

We have shown that in some cases all transpositions and square transpositions of zeroes of $q$ are contained in $\pi_{1}\left(Q_{\lambda}^{0},(M, q)\right)$. However, for $\lambda$ where many but not all points are of equal weight, Theorem 4.14 and Corollary 4.15 imply that the 
kernel $\operatorname{ker}\left(\pi_{1}\left(\operatorname{Sym}_{g}^{\lambda}\right) \rightarrow H_{1}(M, \mathbb{Z})\right)$ is generated by transpositions, null $\rho_{r}$ and $i-$ commutators. In some cases we can again use techniques of colliding and breaking apart points from Section 5 to show that these last two types of elements are in $\pi_{1}\left(\mathcal{Q}_{\lambda}^{0},(M, q)\right)$.

Let $l_{1}, \ldots, l_{2 g}$ be standard generators of $\pi_{1}(M), \lambda$ of length $n$, and $\left(p_{1}, \ldots, p_{n}\right)$ a base point for $B_{\lambda}$. Recall that in Section 3 and Section 4 we did not explicitly define the $\rho_{i r}, 1 \leq i \leq n, 1 \leq r \leq 2 g$, we only stated that one of the generators of $B_{\lambda}$ must be a loop corresponding to $p_{i}$ moving around $l_{r}$. There are infinitely many choices of such a loop, differing by various products of transpositions.

Lemma 7.1 Let $\mathcal{Q}_{\lambda_{1}}=\mathcal{Q}_{g}\left(k_{1}, k_{2}, \ldots, k_{n}\right)$ contain all of its transpositions, and suppose $\sum_{i=1}^{l} k_{i}=\sum_{j=l+1}^{m} k_{j}$. Further suppose there exists a degeneration of $\mathcal{Q}_{\lambda_{1}}^{0}, \mathcal{Q}_{\lambda_{2}}^{0}:=\mathcal{Q}_{g}^{0}\left(k_{1}+\cdots+k_{l}, k_{l+1}+\cdots+k_{m}, k_{m+1}, \ldots, k_{n}\right) \ni(M, q)$, and $\pi_{1}\left(\mathcal{Q}_{\lambda_{2}}^{0},(M, q)\right)$ contains all transpositions of the two newly formed points. Define $\alpha(1), \alpha(2), \ldots, \alpha(l)$ to all equal 1 , and $\alpha(l+1), \ldots, \alpha(m)$ to all equal -1 . Then for any $\left(M^{\prime}, q^{\prime}\right) \in \mathcal{Q}_{\lambda_{1}}$, for any choice of $\rho_{1 r}, \ldots, \rho_{n r}$, for any $i_{1}, \ldots, i_{m}$ such that $k_{i_{s}}=k_{s}, 1 \leq s \leq m$, and for any $\sigma \in S_{m}, \rho_{i_{\sigma(1)} r}^{\alpha(\sigma(1))} \cdots \rho_{i_{\sigma(m)} r}^{\alpha(\sigma(m))} \in \pi_{1}\left(\mathcal{Q}_{\lambda_{1}}^{0},\left(M^{\prime}, q^{\prime}\right)\right)$.
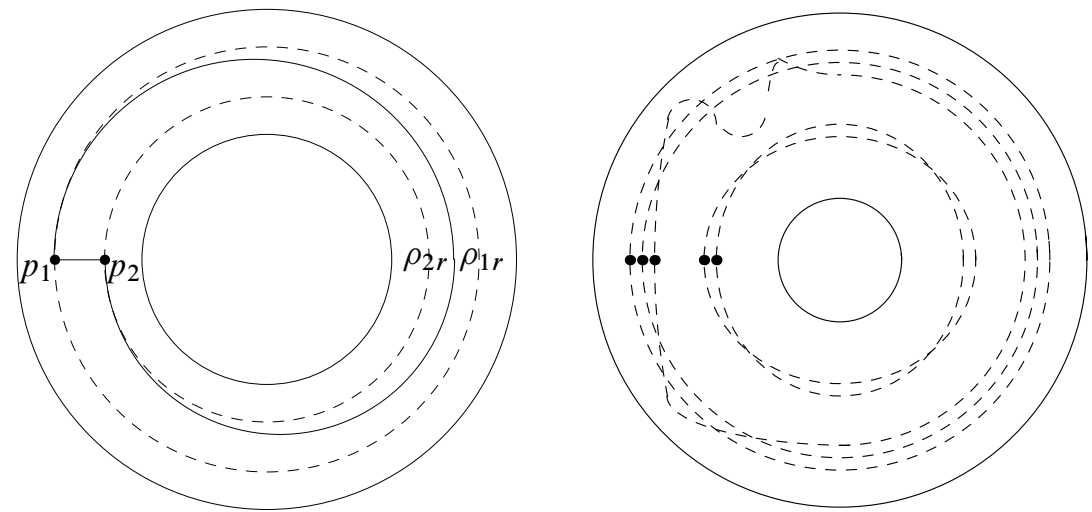

Figure 7: On the left, transposing $p_{1}, p_{2}$ along both the solid curves is equivalent to $\rho_{1 r}^{ \pm} \rho_{2 r}^{\mp}$. On the right, surgering give us one possible choice of $\rho_{i r}$ from the original two.

Proof To prove the lemma we first show that there exist explicit $\rho_{1 r}, \ldots, \rho_{n r}$ such that $\rho:=\rho_{1 r} \cdots \rho_{l r} \rho_{(l+1) r}^{-1} \cdots \rho_{m r}^{-1} \in \pi_{1}\left(\mathcal{Q}_{\lambda},\left(M^{\prime}, q^{\prime}\right)\right)$. We then pick any other choice of generators, $\rho_{1 r}^{\prime}, \ldots, \rho_{n r}^{\prime}$, any $i_{1}, \ldots, i_{m}$ (not necessarily distinct) such that $k_{i_{s}}=k_{s}$, and any permutation $\sigma \in S_{m}$. We define $\rho^{\prime}:=\rho_{i_{\sigma(1)}}^{\alpha} \alpha(\sigma(1)) \cdots \rho_{i_{\sigma(m) r}}^{\alpha(\sigma(m))}$. We show that $\rho^{\prime}$ differs from $\rho$ only by a product of transpositions and is thus also in $\pi_{1}\left(\mathcal{Q}_{\lambda},\left(M^{\prime}, q^{\prime}\right)\right)$. 
Label the two zeroes of $q$ of order $k_{1}+k_{2}+\cdots+k_{l}$ as $p_{1}$ and $p_{2}$. For any $r$ pick two edges, $e$ and $e^{\prime}$, between $p_{1}, p_{2}$ such that $e \cup e^{\prime}$ is homotopic to $l_{r}$ and $e \cap e^{\prime}=\left\{p_{1}, p_{2}\right\}$, as in the left of Figure 7. By assumption $\sigma_{e}, \sigma_{e^{\prime}} \in \pi_{1}\left(\mathcal{Q}_{\lambda_{2}}^{0},(M, q)\right)$. Further, defining $\rho_{1 r}$ and $\rho_{2 r}$ as in the left of Figure 7, $\sigma_{e} \sigma_{e^{\prime}}$ is homotopic to $\rho_{1 r} \rho_{2 r}^{-1}$. Thus $\rho_{1 r} \rho_{2 r}^{-1} \in \pi_{1}\left(\mathcal{Q}_{\lambda_{2}}^{0},(M, q)\right)$.

Now pick an explicit loop of surfaces in the homotopy class $\rho_{1 r} \rho_{2 r}^{-1}, \eta:[0,1] \rightarrow \mathcal{Q}_{\lambda_{2}}^{0}$, such that $\eta(0)=\eta(1)=(M, q)$.

Surger every $\eta(t)$ by one of the surgeries of Lemmas 5.2-5.3 such that the new trajectory created is horizontal, and $\delta_{t}$ varies continuously, with $\delta_{0}=\delta_{1}$. This creates a new curve of surfaces, $\gamma:[0,1] \rightarrow \mathcal{Q}_{g},\left(\gamma([0,1])\right.$ is either in $\mathcal{Q}_{\lambda_{1}}$ or in some "intermediate" stratum between $\mathcal{Q}_{\lambda_{1}}$ and $\mathcal{Q}_{\lambda_{2}}$ ) such that either $\gamma(0)=\gamma(1)$ or $\gamma(0)$ differs from $\gamma(1)$ in that the newly formed trajectories' directions differ by a multiple of $\pi$. In the latter case, label the direction of the trajectory formed by $\gamma(0)$ as $0^{\circ}$, so the one formed by $\gamma(1)$ will be in the direction $k \pi, 1 \leq k \leq k_{1}+k_{2}+\cdots+k_{l}+2$. Consider the curve of surfaces $\alpha:[0, k \pi] \rightarrow \mathcal{Q}_{g}$ created by surgering $\eta(0)$ with respect to $\delta_{0}, t$. Concatenating $\gamma$ with $\alpha$ gives us a closed curve of surfaces, again either in $\mathcal{Q}_{\lambda_{1}}$ or an intermediate stratum.

Repeating the above process multiple times if necessary, we get a loop of surfaces $\eta^{\prime}:[0,1] \rightarrow \mathcal{Q}_{\lambda_{1}}$, and define $\left(M^{\prime}, q^{\prime}\right)=\eta^{\prime}(0)$. Let $p_{1}(t), \ldots, p_{m}(t)$ be the zeroes of $\eta^{\prime}(t)$ of order $k_{1}, \ldots, k_{m}$ formed from the surgery. We have created an element of $\pi_{1}\left(\mathcal{Q}_{\lambda_{1}},\left(M^{\prime}, q^{\prime}\right)\right)$ such that each of $p_{1}, \ldots, p_{m}$ follows a path homotopic to $l_{r}^{ \pm 1}$. Thus we may choose to define $\rho_{1 r}, \ldots, \rho_{m r}$ in such a way that $\rho:=\rho_{1 r} \cdots \rho_{l r} \rho_{(l+1) r}^{-1} \cdots \rho_{m r}^{-1} \in \pi_{1}\left(\mathcal{Q}_{\lambda_{1}},\left(M^{\prime}, q^{\prime}\right)\right)$.

Now let $\sigma$ be an element of $S_{m}$ and consider $\rho_{\sigma}:=\rho_{\sigma(1) r}^{\alpha(\sigma(1))} \cdots \rho_{\sigma(m) r}^{\alpha(\sigma(m))}$. The product $\rho_{\sigma} \rho^{-1}$ is an element of $B_{\lambda}$, but since $p_{1}(0), \ldots, p_{m}(0)$ all return to themselves under $\rho_{\sigma} \rho^{-1}$ and the other zeroes of $\left(M^{\prime}, q^{\prime}\right)$ follow constant paths, we may view $\rho_{\sigma} \rho^{-1}$ as an element of $S B_{m}$. It is a theorem of Goldberg (see Birman [1, Section 1.4]) that for any genus $g$ topological surface, $M_{g}$, the following sequence is short exact:

$$
1 \rightarrow S B_{m}(D) \rightarrow S B_{m}\left(M_{g}\right) \stackrel{\psi}{\rightarrow} \prod_{i=1}^{m} \pi_{1}\left(M_{g}\right) \rightarrow 1
$$

where $D \subset M_{g}$ is an open disc containing the $m$ marked points that are the basepoint of $S B_{m}$. Since the path traveled by each of the zeroes of $\left(M^{\prime}, q^{\prime}\right)$ under $\rho_{\sigma} \rho^{-1}$ is trivial in $\pi_{1}\left(M^{\prime}\right), \rho_{\sigma} \rho^{-1}$ is in the kernel of $\psi$. Thus, it may be written as a product of the generators of $S B_{m}(D)$, which are square transpositions. We have assumed $\pi_{1}\left(\mathcal{Q}_{\lambda},\left(M^{\prime}, q^{\prime}\right)\right)$ contains all (square) transpositions, so $\rho_{\sigma} \rho^{-1} \in \pi_{1}\left(\mathcal{Q}_{\lambda},\left(M^{\prime}, q^{\prime}\right)\right)$ and therefore $\rho_{\sigma} \in \pi_{1}\left(\mathcal{Q}_{\lambda},\left(M^{\prime}, q^{\prime}\right)\right)$. 
Similarly define $\rho_{1 r}^{\prime}, \rho_{2 r}^{\prime}, \ldots, \rho_{n r}^{\prime}$ to be a different choice of generators, and define $\rho^{\prime}:=\rho_{1 r}^{\prime} \cdots \rho_{l r}^{\prime} \rho_{(l+1) r}^{\prime} \cdots \rho_{m r}^{\prime}$. Again $\rho^{\prime} \rho^{-1}$ is in the kernel of $\psi$ and thus $\rho^{\prime} \in$ $\pi_{1}\left(\mathcal{Q}_{\lambda},\left(M^{\prime}, q^{\prime}\right)\right)$.

Finally suppose we have $p_{i_{1}}, \ldots, p_{i_{m}}$ (not necessarily distinct) such that $k_{i_{s}}=k_{s}$, $1 \leq s \leq m$, and define $\rho_{i}:=\rho_{i_{1}} \cdots \rho_{i_{l}} \rho_{i_{l+1}}^{-1} \cdots \rho_{i_{m}}^{-1}$. Either $s=i_{s}$ and $\rho_{s r}^{ \pm} \rho_{i_{s} r}^{\mp}=1$ or $\rho_{s r}^{ \pm} \rho_{i_{s} r}^{\mp}$ may be written as a product of two transpositions, as in the first paragraph of this proof. Then the following is in $\pi_{1}\left(\mathcal{Q}_{\lambda},\left(M^{\prime}, q^{\prime}\right)\right)$ :

$$
\beta:=\rho_{1 r} \rho_{i_{1} r}^{-1} \rho_{2 r} \rho_{i_{2} r}^{-1} \cdots \rho_{l r} \rho_{i_{l} r}^{-1} \rho_{(l+1) r}^{-1} \rho_{i_{l+1} r} \cdots \rho_{m r}^{-1} \rho_{i_{m} r}
$$

As above $\rho_{i} \beta \rho^{-1}$ is in the kernel of $\psi$ and is therefore in $\pi_{1}\left(\mathcal{Q}_{\lambda},\left(M^{\prime}, q^{\prime}\right)\right)$. This then implies $\rho_{i} \in \pi_{1}\left(\mathcal{Q}_{\lambda},\left(M^{\prime}, q^{\prime}\right)\right)$.

A combination of the above implies the lemma for the specific choice of $\left(M^{\prime}, q^{\prime}\right)$, surgered from $(M, q)$. However, since fundamental groups with different base points are isomorphic, the same will be true for any element of $\mathcal{Q}_{\lambda_{1}}$.

Lemma 7.1 shows that under certain degeneracy conditions it is possible to construct any null $\rho_{r}$ involving points of certain weights. Notice that when $l, m=1$ the lemma implies that if $\pi_{1}\left(\mathcal{Q}_{\lambda_{1}}^{0},\left(M^{\prime}, q^{\prime}\right)\right)$ contains all of its transpositions, it also contains all null $\rho_{r}$ involving two points of equal weights.

Proposition 7.2 Suppose that $g>2$ and $\lambda_{1}=\left(1^{a}, k_{1}, \ldots, k_{n}\right)$ with exponent $a>$ $\max \left\{g+4, k_{1}, \ldots, k_{n}\right\}$, and additionally assume that all $k_{i}$ are even and there exists $s, t, 1 \leq s, t \leq n$ such that $k_{s}=k_{t}$. Then for any $\left(M^{\prime}, q^{\prime}\right)$, all null $\rho_{r}$ are contained in $\pi_{1}\left(Q_{\lambda_{1}},\left(M^{\prime}, q^{\prime}\right)\right)$.

Proof By Theorem 2.7 there is only one connected component of $\mathcal{Q}_{\lambda_{1}}$.

Let $\left(M^{\prime}, q^{\prime}\right) \in \mathcal{Q}_{\lambda_{1}}$ with $p_{1}, \ldots, p_{a}$ the zeroes of $q$ of order 1 . By Proposition 6.6 $\pi_{1}\left(\mathcal{Q}_{\lambda_{1}},\left(M^{\prime}, q^{\prime}\right)\right)$ contains all of its transpositions; by Lemma 7.1 it contains any $\rho_{j r} \rho_{l r}^{-1}, 1 \leq j, l \leq a, 1 \leq r \leq 2 g$.

For each $k_{i}, 1 \leq i \leq n$ and each $r$ we construct a null $\rho_{r}$ consisting of $p_{a+i}$ and $k_{i}$ zeroes of order 1 . These, combined with the $\rho_{j r} \rho_{l r}^{-1}$ where $k_{j}=k_{l}=1$, generate all null $\rho_{r}$. We do this in two cases, when $k_{i} \neq 2$ and when $k_{i}=2$.

For $k_{i} \neq 2$, let $\mathcal{Q}_{\lambda_{2}}=\mathcal{Q}_{g}\left(1^{a-k_{i}}, k_{1}, \ldots, k_{i}^{2}, \ldots, k_{n}\right)$ and notice that by Lemma 5.2 and Lemma 5.3, $\lambda_{1}>\lambda_{2}$ (this is not true if $k_{i}=2$ ). Since $k_{i}$ is even there exists a component of $\mathcal{Q}_{\lambda_{2}}, \mathcal{Q}_{\lambda_{2}}^{0}$, that contains a hyperelliptic element, $(M, q)$, for which both zeroes of order $k_{i}$ are at branch points. By Lemma 6.3, $\pi_{1}\left(\mathcal{Q}_{\lambda_{2}}^{0},(M, q)\right)$ contains all transpositions of the two zeroes of order $k_{i}$. Therefore Lemma 7.1 implies any null $\rho_{r}$ 
consisting of a single zero of order $k_{i}$ moving around $l_{r}$ and $k_{i}$ zeroes of order 1 moving around $l_{r}^{-1}$ is in $\pi_{1}\left(\mathcal{Q}_{\lambda_{1}},\left(M^{\prime}, q^{\prime}\right)\right)$.

If $k_{i}=2$ we let $\mathcal{Q}_{\lambda_{2}}=\mathcal{Q}_{g}\left(1^{a-6}, 4^{2}, k_{1}, \ldots, \hat{k}_{i}, \ldots, k_{n}\right)$ and note that $\lambda_{1}>\lambda_{2}$. By the same argument as in the previous paragraph there exists $(M, q) \in \mathcal{Q}_{\lambda_{2}}$ such that $\pi_{1}\left(\mathcal{Q}_{\lambda_{2}},(M, q)\right)$ contains each of the $2 g$ null $\rho_{r}$ involving only the two zeroes of order 4 . Thus we get the null $\rho_{r}$ involving a zero of order 2 and 2 zeroes of order 1 moving one way around $l_{r}$, and 4 zeroes of order 1 moving the other. We compose and cancel with null $\rho_{r}$ involving points of order 1 to get a null $\rho_{r}$ with a zero of order 2 moving around $l_{r}$ and two zeroes of order 1 moving around $l_{r}^{-1}$.

This gives us any null $\rho_{r}$ consisting of a single zero of higher order moving one way around $l_{r}$ and zeroes of order 1 moving the other. These and the $\rho_{j r} \rho_{l r}^{-1}$ where $k_{j}=k_{l}=1$ generate all null $\rho_{r}$.

Notice that there are strata of the form specified in Proposition 6.6 for which there exist null $\rho_{r}$ to which Lemma 7.1 does not apply. For example, in $\mathcal{Q}_{10}\left(1^{16}, 20\right)$ we can have a null $\rho_{r}$ consisting of the point of order 20 moving one way around $l_{r}$ and 20 points of order 1 moving the other, but since there are not 20 distinct points of order 1 we cannot collide them to use the technique of Lemma 7.1.

Finally we would like to consider when $i$-commutators are contained in $\pi_{1}\left(\mathcal{Q}_{\lambda}\right)$.

Proposition 7.3 Let $\lambda=\left(1^{a}, k_{1}, \ldots, k_{n}\right)$, where $a$ and the $k_{i}$ are as in Proposition $7.2,1 \leq i \leq n$. Then for any $(M, q) \in \mathcal{Q}_{\lambda}$, any $i$-commutator of a point not of weight one is in $\pi_{1}\left(Q_{\lambda},(M, q)\right)$.

Proof Let $p_{1}, p_{2}, \ldots, p_{a}$ be the zeroes of $q$ of weight 1 , and $p_{a+1}, \ldots, p_{a+n}$ the zeroes of weight $k_{1}, \ldots, k_{n}$, where $k_{j}$ is by assumption is less than $a, 1 \leq j \leq n$. Recall we defined an $i$-commutator to be an element of the commutator of $\left\langle\rho_{a+i, r}, \kappa_{a+i, j}\right\rangle$, $1 \leq r \leq 2 g, a+i<j \leq n$. Thus it suffices to show that $\pi_{1}\left(Q_{\lambda},(M, q)\right)$ contains all elements of the form $g^{-1} h^{-1} g h$, where $g$ and $h$ are arbitrary words in the $\rho_{a+i, r}$ and $\kappa_{a+i, j}$.

By Proposition $7.2 \pi_{1}\left(\mathcal{Q}_{\lambda},(M, q)\right)$ contains all of its null $\rho_{r}$. Thus for any $i, 1 \leq i \leq n$, the following is also in $\pi_{1}\left(Q_{\lambda},(M, q)\right)$ :

$$
\left(\rho_{1 r} \cdots \rho_{k_{i} r} \rho_{a+i, r}^{-1}\right)\left(\rho_{1 s} \cdots \rho_{k_{i} s} \rho_{a+i, s}^{-1}\right)\left(\rho_{1 r}^{-1} \cdots \rho_{k_{i} r}^{-1} \rho_{a+i, r}\right)\left(\rho_{1 s}^{-1} \cdots \rho_{k_{i} s}^{-1} \rho_{a+i, s}\right)
$$

For $k \neq j$ and $l_{r} \cap l_{s}=\varnothing, \rho_{k r}$ commutes with $\rho_{j s}, 1 \leq j, k \leq a+n$. If $k \neq j$ but $l_{r} \cap l_{s}=1$ then $\rho_{k r} \rho_{j s}=\rho_{j s} \rho_{k r} \kappa_{e}$ where $\kappa_{e}$ is a square transposition of $p_{k}, p_{j}$, defined appropriately with respect to $\rho_{j s}, \rho_{k r}$. For example, in Figure 8 we have two 


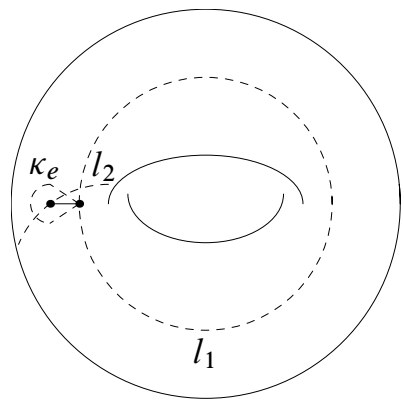

Figure 8: $\rho_{11}, \rho_{22}$, and $\kappa_{e}$ defined on a torus with two marked points. The solid line is $e$.

points moving around the cycles $l_{1}, l_{2}$ on a torus and an explicitly defined $\kappa_{e}$, with $\rho_{11} \rho_{22}=\rho_{22} \rho_{11} \kappa_{e}$.

Thus if $l_{r} \cap l_{s}=\varnothing$, (8) is equal to

$$
\left(\rho_{1 r} \cdots \rho_{k_{i} r} \rho_{1 s} \cdots \rho_{k_{i} s} \rho_{1 r}^{-1} \cdots \rho_{k_{i} r}^{-1} \rho_{1 s}^{-1} \cdots \rho_{k_{i} s}^{-1}\right)\left(\rho_{a+i, r}^{-1} \rho_{a+i, s}^{-1} \rho_{a+i, r} \rho_{a+i, s}\right) .
$$

Notice that the maximal value for $n$ is $(4 g-4-(g+5)) / 2$, and this implies that $a \geq g+5 \geq(3+\sqrt{9+8(2 g+n-2)}) / 2$. The first of the two elements in parentheses in (9) is in the kernel of $\mathrm{AJ}_{*}: \pi_{1}\left(\operatorname{Sym}^{a}\left(M_{n}\right)\right) \rightarrow H_{1}(M, \mathbb{Z})$, so we may apply Corollary 4.6 to show that it can be written as a product of transpositions. Proposition 6.6 says that $\pi_{1}\left(\mathcal{Q}_{\lambda},(M, q)\right)$ contains all of its transpositions; thus the element on the left is in $\pi_{1}\left(\mathcal{Q}_{\lambda},(M, q)\right)$. This in turn implies $\rho_{a+i, r} \rho_{a+i, s} \rho_{a+i, r}^{-1} \rho_{a+i, s}^{-1} \in \pi_{1}\left(\mathcal{Q}_{\lambda},(M, q)\right)$.

If $l_{r} \cap l_{s}=1$ then (8) is equal to (9) except that the first element in parentheses will contain some additional $\kappa_{e}$ 's. However it will still be in the kernel of $\mathrm{AJ}_{*}$ and again $\rho_{a+i, r} \rho_{a+i, s} \rho_{a+i, r}^{-1} \rho_{a+i, s}^{-1} \in \pi_{1}\left(\mathcal{Q}_{\lambda},(M, q)\right)$. The above argument is easily generalized to show that the commutators of arbitrary words in the $\rho_{a+i, r}, 1 \leq r \leq 2 g$, are in $\pi_{1}\left(Q_{\lambda},(M, q)\right)$.

Similarly, for $p_{l}$ a zero of higher order and $e^{\prime}$ an edge between $p_{i}$ and $p_{l}$, we would like to show that $\kappa_{e^{\prime}}^{-1} \rho_{a+i, r}^{-1} \kappa_{e^{\prime}} \rho_{a+i, r}$ is in $\pi_{1}\left(\mathcal{Q}_{\lambda},(M, q)\right)$, so we consider the following:

$$
\kappa_{e^{\prime}}^{-1}\left(\rho_{1 r} \cdots \rho_{k_{i} r} \rho_{a+i, r}^{-1}\right) \kappa_{e^{\prime}}\left(\rho_{1 r}^{-1} \cdots \rho_{k_{i} r}^{-1} \rho_{a+i, r}\right)
$$

By assumption both $\kappa_{e^{\prime}}^{ \pm 1}$ and the elements in parentheses are in $\pi_{1}\left(\mathcal{Q}_{\lambda},(M, q)\right)$, so the whole element in (10) is. Since $\kappa_{e^{\prime}}$ commutes with $\rho_{1 r}, \ldots, \rho_{k r}$, commuting the $\rho_{1 r}, \ldots, \rho_{1 k}$ as from (8) to (9) gives us the desired result. A combination of this argument and the above shows that the commutators of arbitrary words in the $\rho_{a+i, r}$ and $\kappa_{a+i, j}$ are in $\pi_{1}\left(\mathcal{Q}_{\lambda},(M, q)\right)$. 


\section{Conclusion}

We summarize by answering the question of when $\operatorname{ker}\left(\mathrm{AJ}_{*}: \pi_{1}\left(\operatorname{Sym}_{g}^{\lambda}\right) \rightarrow H_{1}(M, \mathbb{Z})\right)$ is equal to $\operatorname{im}\left(i_{*}: \pi_{1}\left(\mathcal{Q}_{\lambda}\right) \rightarrow \pi_{1}\left(\operatorname{Sym}_{g}^{\lambda}\right)\right)$.

Theorem 8.1 Let $\lambda=\left(1^{a}, k_{1}, \ldots, k_{n}\right)$ with $a>\max \left\{g+5, k_{1}, \ldots, k_{n}\right\}$, all $k_{i}$ even, and some $k_{i}=k_{j}, 1 \leq i<j \leq n$. Then $\operatorname{im}\left(i_{*}\right)=\operatorname{ker}\left(\mathrm{AJ}_{*}\right)$.

Proof Proposition 4.1 implies $\operatorname{im}\left(i_{*}: \pi_{1}\left(\mathcal{Q}_{\lambda}\right) \rightarrow \pi_{1}\left(\operatorname{Sym}_{g}^{\lambda}\right)\right) \subset \operatorname{ker}\left(\mathrm{AJ}_{*}\right)$. By Theorem 4.14 and Corollary $4.15 \operatorname{ker}\left(\mathrm{AJ}_{*}\right)$ is generated by transpositions, square transpositions, null $\rho_{r}$ and in some cases $i$-commutators. Proposition 6.6, Proposition 7.2, and Proposition 7.3 show that all of these elements are in $\operatorname{im}\left(i_{*}: \pi_{1}\left(\mathcal{Q}_{\lambda}\right) \rightarrow \pi_{1}\left(\operatorname{Sym}_{g}^{\lambda}\right)\right)$

In [2], Copeland shows a similar result for $g>2$ and $\lambda=\left(1^{4 g-4}\right)$. His techniques are somewhat different and rely on the fact that in the top stratum one may interpolate two quadratic differentials and expect the result to be in the same stratum.

Thus, for certain $\lambda$ we have constructed $i_{*}\left(\pi_{1}\left(\mathcal{Q}_{\lambda}\right)\right)$. Of course, we are actually interested in $\pi_{1}\left(\mathcal{Q}_{\lambda}\right)$ and would thus like to determine the kernel of $i_{*}$. However, it may be difficult to say anything about this kernel.

Acknowledgments I would like to thank P Seidel, H Masur, D Canary and especially J Copeland for illuminating conversations and helpful comments. I would also like to thank the referee for a careful reading of the paper and a number of helpful suggestions.

\section{References}

[1] J S Birman, Braids, links, and mapping class groups, Annals of Math. Studies 82, Princeton Univ. Press (1974) MR0375281

[2] D J Copeland, Monodromy of the Hitchin map over hyperelliptic curves, Int. Math. Res. Not. (2005) 1743-1785 MR2172340

[3] A Eskin, H Masur, A Zorich, Moduli spaces of abelian differentials: the principal boundary, counting problems, and the Siegel-Veech constants, Publ. Math. Inst. Hautes Études Sci. (2003) 61-179 MR2010740

[4] E Fadell, L Neuwirth, Configuration spaces, Math. Scand. 10 (1962) 111-118 MR0141126

[5] P Griffiths, J Harris, Principles of algebraic geometry, Wiley Classics Library, Wiley, New York (1994) MR1288523 Reprint of the 1978 original

[6] M Kontsevich, A Zorich, Connected components of the moduli spaces of Abelian differentials with prescribed singularities, Invent. Math. 153 (2003) 631-678 MR2000471 
[7] H V Kronk, R D Ringeisen, A T White, On 2-cell imbeddings of complete n-partite graphs, Colloq. Math. 36 (1976) 295-304 MR0460164

[8] E Lanneau, Hyperelliptic components of the moduli spaces of quadratic differentials with prescribed singularities, Comment. Math. Helv. 79 (2004) 471-501 MR2081723

[9] H Masur, J Smillie, Hausdorff dimension of sets of nonergodic measured foliations, Ann. of Math. (2) 134 (1991) 455-543 MR1135877

[10] H Masur, A Zorich, Multiple saddle connections on flat surfaces and the principal boundary of the moduli spaces of quadratic differentials, Geom. Funct. Anal. 18 (2008) 919-987 MR2439000

[11] G P Scott, Braid groups and the group of homeomorphisms of a surface, Proc. Cambridge Philos. Soc. 68 (1970) 605-617 MR0268889

[12] W A Veech, The Teichmüller geodesic flow, Ann. of Math. (2) 124 (1986) 441-530 MR866707

[13] K C Walker, Connected components of the strata of quadratic differentials over the Teichmüller space, Geom. Dedicata 142 (2009) 47-60 MR2545455

Center for Communications Research

4320 Westerra Court, San Diego, CA 92121

kcwalk@ccrwest.org

Proposed: Benson Farb

Received: 23 May 2008

Seconded: Danny Calegari, Joan Birman

Revised: 19 November 2009 